\title{
Performance Analysis of Bullet Trajectory Estimation: Approach, Simulation, and Experiments
}

\author{
Lawrence C. $\mathrm{Ng}^{*}$ \\ Thomas J. Karr
}

November 8, 1994

Lawrence Livermore National Laboratory

L-278

P.O. Box 808

Livermore, CA 94551

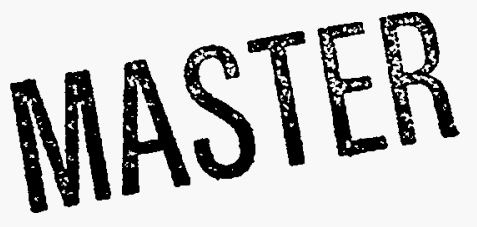

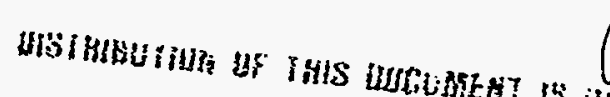




\section{DISCLAIMER}

This report was prepared as an account of work sponsored by an agency of the United States Government. Neither the United States Government nor. any agency thereof, nor any of their employees, make any warranty, express or implied, or assumes any legal liability or responsibility for the accuracy, completeness, or usefulness of any information, apparatus, product, or process disclosed, or represents that its use would not infringe privately owned rights. Reference herein to any specific commercial product, process, or service by trade name, trademark, manufacturer, or otherwise does not necessarily constitute or imply its endorsement, recommendation, or favoring by the United States Government or any agency thereof. The views and opinions of authors expressed herein do not necessarily state or reflect those of the United States Government or any agency thereof. 


\section{DISCLAIMER}

Portions of this document may be illegible in electronic image products. Images are produced from the best available original document. 


\begin{abstract}
This paper describes an approach to estimate a bullet's trajectory from a time sequence of angles-only observations from a high-speed camera, and analyzes its performance. The technique is based on fitting a ballistic model of a bullet in flight along with unknown source location parameters to a time series of angular observations. The theory is developed to precisely reconstruct, from firing range geometry, the actual bullet trajectory as it appeared on the focal plane array and in real space. A metric for measuring the effective trajectory track error is also presented. Detailed Monte-Carlo simulations assuming different bullet ranges, shot-angles, camera frame rates, and angular noise show that angular track error can be as small as $100 \mu \mathrm{rad}$ for a $2 \mathrm{mrad} /$ pixel sensor. It is also shown that if actual values of bullet ballistic parameters were available, the bullet's source location variables, and the angles of flight information could also be determined.
\end{abstract}

*Larry $\mathrm{Ng}$ is with the Engineering Research Division in Electronics Engineering matrixed to the Advanced Technology Program. Tom Karr is the Principal Investigator of the project and resides in the Special Studies Division. 


\section{Table of Contents}

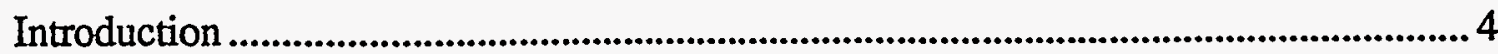

Problem Formulation (no gravity) .......................................................................... 4

AZEL State Vector Estimation .......................................................................................... 6

Extension To Out of Plane Trajectory ..................................................................

Extension to No Muzzle Flash Detection ........................................................... 8

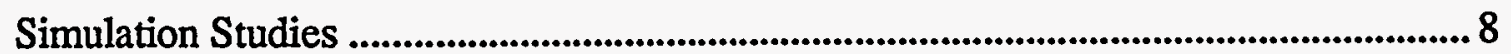

AZEL Track Estimation with Muzzle Flash Detection .........................................9

Metric for Effective Track Error ............................................................................. 11

Parametric Sensitivity Study of Track Errors ......................................................... 12

AZEL Track Estimation with No Muzzle Flash Detection..................................... 14

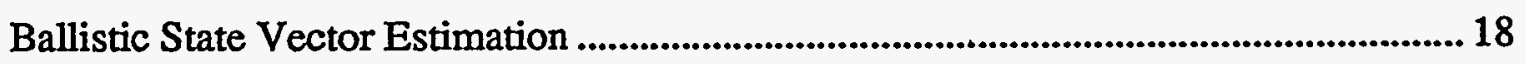

Error Analysis of Source Location Parameters ........................................................ 19

Experimental Setup …........................................................................................................... 21

Bullet Trajectory Reconstruction .............................................................................22

Bullet Tracking Experiments and Performance Validation ..............................................22

Practical Considerations for Real-time Source Location ........................................25

Summary and Conclusions.....................................................................................................26

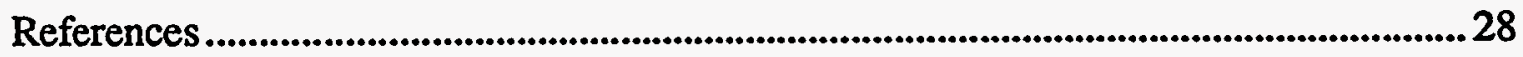

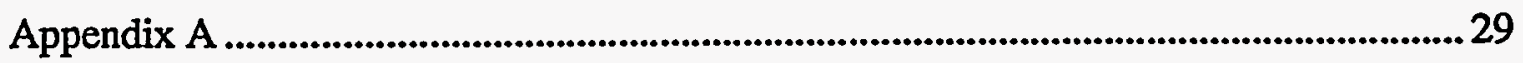

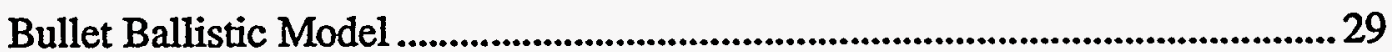

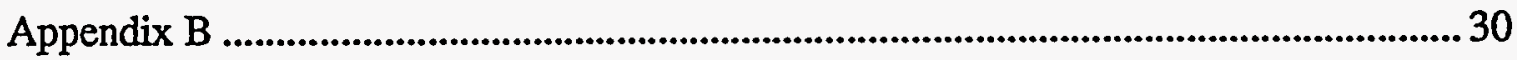

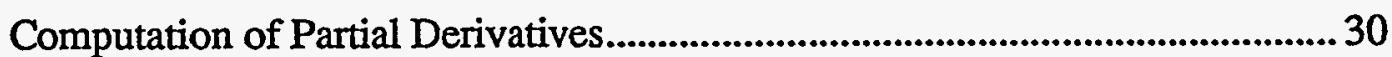

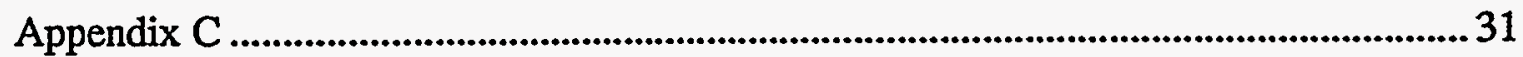

Survey Map at LLNL Site 300 Firing Range........................................................ 31

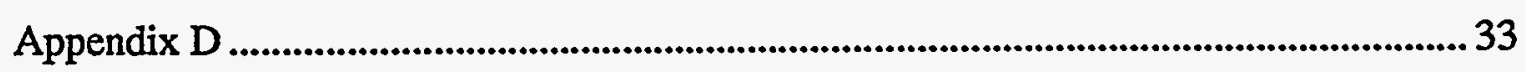

Bullet Trajectory Reconstruction from Firing Range Data.................................... 33 


\section{Introduction}

Estimation of target trajectory from a time sequence of angles-only observations has long been studied by the Navy in the context of submarine target motion analysis [1-5]. A key issue in the analysis is the problem of observability [6]. Observability measures the ability to process a set of observations in order to estimate unambiguously the unknown target parameters such as position and velocity. For the submarine problem it was found that under a constant-velocitytarget assumption, observability is achieved by inducing bearing accelerations from platform maneuvers.

The bullet tracking problem differs from the submarine target motion analysis problem in three important aspects: (1) the sensor platform moves very little during the observed bullet time of flight, and (2) the bullet undergoes a tremendous deceleration due to aerodynamic drag, and (3) the bullet travels along a nearly straight line path over the short observation time interval of several $100 \mathrm{~ms}$. Thus for the purposes of track estimation, neglecting gravity often is acceptable. [Note: We analyze the trajectory estimation problem including gravity in a forthcoming paper.]

This study analyses the source location problem (i.e., where the bullet was fired), the shot angle (where the bullet is going), and the time of fire (when the bullet leaves the gun muzzle). A metric was developed to measure the effective error of the estimated bullet trajectory. Detailed Monte-Carlo simulations were conducted to assess its performance for different firing ranges, shot-angles, camera frame rates, and angular noise. This study also found that full observability can be achieved if knowledge of bullet muzzle velocity and ballistic coefficient is available.

This study also investigates the performance (accuracy) of the resulting trajectory reconstruction from firing range geometry as it appears (1) on the focal plane array, and (2) actual geometric space. We will call the first problem azimuth elevation (AZEL) state vector estimation, and the latter as ballistic state vector estimation.

\section{Problem Formulation (no gravity)}

We choose to analyse a planar case first. We also neglect the effect of gravity, so the trajectory stays on a horizontal plane. As shown in Figure 1, we assume at time $t_{0}$, a bullet was fired at position $\left(\mathrm{x}_{0}\right.$, yo) with muzzle velocity $v_{0}$, and at a shotangle $\phi$, with respect to (w.r.t) the $Y$ axis. A camera, located at the axis origin, with a given field-of-view (FOV), will observe a time sequence of bullet positions.

Furthermore, let $s(t)$ be the bullet position measured from the point of fire at time $t$ and $v(t)$ be the velocity. Also the bullet is located at angle $\theta(t)$, w.r.t. the $Y$ axis pointing at the firing point. Discrete angular measurements of the bullet in flight were collected as $\theta_{k}$ at time $t_{k} ; k=0,1,2, \ldots, N$. Note, in our notation, $\theta_{0}$ is the muzzle flash, and $\theta_{1}$ and $\theta_{N}$ correspond to the first and last bullet detections.

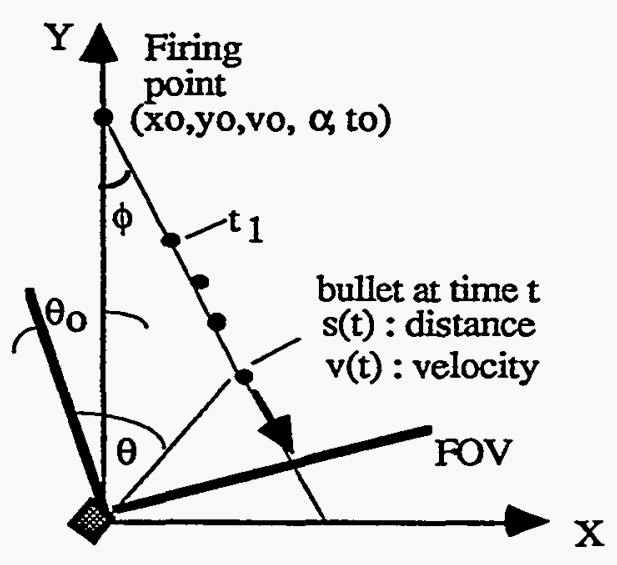
position

Figure 1 Planar bullet camera geometry
Camera 
Our problem is the following one. Based on a set of measurements

$$
Z_{\mathrm{N}}=\left[\theta_{0}, \theta_{1}, \ldots \theta_{\mathrm{N}}\right]^{\mathrm{T}},
$$

a column vector, we want to estimate the six unknowns given by

$$
\begin{aligned}
& x_{0}=x \text { coordinate of bullet initial location, } \\
& y_{0}=y \text { coordinate of bullet initial location, } \\
& v_{0}=\text { muzzle velocity } \\
& \phi \quad=\text { shot angle, } \\
& \alpha=\text { bullet's ballistic drag coefficient (or inverse of ballistic coefficient), and } \\
& t_{0}=\text { time of fire. }
\end{aligned}
$$

When a muzzle flash is detected, then both $t_{0}$ and $x_{0}$ are known and can be eliminated from the parameter estimation problem; otherwise one must estimate all six parameters. The estimation problem can be formulated and numerically solved as a least mean squares problem as follows:

At any time $t=t k$, the angular measurement is related to the position and velocity state of the bullet by the relation:

$$
\begin{aligned}
\theta_{k} & =\tan ^{-1}\left(\frac{x\left(t_{k}\right)-x_{o}}{y_{o}-y\left(t_{k}\right)}\right)+\theta_{o} \\
& =\tan ^{-1}\left(\frac{s\left(t_{k}\right) \sin \varphi-x_{o}}{y_{o}-s\left(t_{k}\right) \cos \varphi}\right)+\theta_{o}
\end{aligned}
$$

where

$$
s\left(t_{k}\right)=\frac{1}{\alpha} \ln \left(1+\alpha \mathbf{v}_{o}\left(t_{k}-t_{o}\right)\right),
$$

is the net distance a bullet traveled along the track and is shown in Eq.(A-4) from Appendix A. Thus using Eqs.(2) \& (3) one can rewrite in general the relation between measurement and the unknown parameters as:

$$
\begin{aligned}
\theta_{k} & =h\left(x_{o}, y_{o}, \varphi, \alpha, v_{o}, t_{k}\right)+\theta_{o} ; k=0,1,2,3, \ldots, N \\
& =h\left(\mathbf{x}, t_{k}\right) ;
\end{aligned}
$$

where

$$
\mathbf{x}=[\underbrace{x_{0} y_{0} \varphi t_{0}}_{\mathbf{p}} \underbrace{\alpha \mathbf{v}_{g}}_{\mathrm{w}}]^{T} .
$$

Note in Eq.(4), we further partitioned the parameter vector into position data (p) and weapon data (w). Using Eq.(4) in Eq.(1), we obtain a concise relation between the measurement vector and the unknown parameter vector: 


$$
Z_{N .}=\left[\begin{array}{c}
\theta_{o} \\
\theta_{1} \\
\vdots \\
\theta_{N}
\end{array}\right]=\left[\begin{array}{c}
h\left(\mathbf{x}, t_{o}\right) \\
h\left(\mathbf{x}, t_{1}\right) \\
\vdots \\
h\left(\mathbf{x}, t_{N}\right)
\end{array}\right]=\mathbf{h}_{\mathbf{N}}(\mathbf{x})
$$

Furthermore let $\mathbf{W}_{\mathrm{N}}$ represent the $\mathrm{N}+1$ element noise vector collected from each angular measurement. Then one can formulate the problem into a standard least squares form as:

$$
\mathbf{Z}_{N}=\mathbf{h}_{N}(\mathbf{x})+\mathbf{W}_{N} .
$$

The optimization problem becomes: Find $\mathbf{x}$ to minimize the functional

$$
J(\mathbf{x})=\left(\mathbf{Z}_{N}-\mathbf{h}_{N}(\mathbf{x})\right)^{T}\left(\mathbf{Z}_{N}-\mathbf{h}_{N}(\mathbf{x})\right)
$$

If $\mathbf{x}$ is observable in Eq.(6), that one can solve $\mathbf{x}$ in terms of the measurement vector $\mathbf{Z}_{\mathrm{N}}$ from the minimization process of Eq.(7). For the noiseless case (i.e., perfect measurements) and with muzzle flash detection, a minimum of 4 independent bullet observations would be needed since for this case we only have 4 unknowns. However, because the measurements are noisy, we must solve Eq.(7) using the least mean squares minimization. Note it is well known in the literature that passive angles-only track estimation is difficult to achieve. In fact without gravity Eq.(6) is not fully observable for the bullet tracking problem since neither the sensor nor the bullet deviates from a straight line path over the observation interval. In order to intelligently estimate the source location and the bullet track without gravity, we must make use of appropriate prior knowledge. Thus the real question is what is the minimum set of information we need to supply in order to achieve observability. The following sections document some of our approaches and their experimental validations.

\section{AZEL State Vector Estimation}

In many bullet tracking situations, a muzzle flash is detected in addition to the bullets. However, in most instances bullets are not detected on every frame where they are present. Thus we are interested in the feasibility of reconstructing the azimuth and elevation locations of a bullet as they would appear on the Focal Plane Array (FPA) at each time frame including the frame containing the muzzle flash. Because of our choice of coordinate system in Figure $1, \mathrm{x}_{0}=$ 0 and $t_{0}$ is known. Note also that as measured on the FPA, the precised pixel location of the muzzle flash is not available because it contains many pixels. The unknown parameter vector is given by $\mathbf{x}=\left[y_{o}, \varphi, \alpha, v_{o}\right]$. We choose to solve Eq.(7) using an iterative search approach by linearizing the measurement equation about the estimated parameter state vector. Note for AZEL estimation, the solution for $\mathrm{x}$ is not unique since the observability matrix does not achieve full rank. Specifically, the rate of change of the observed angle with respect to range is proportional to the rate of change of the angle with respect to velocity. There are many possible solutions. For example, if we scale the initial state vector by $\mathrm{x}_{\mathrm{o}}^{\prime}=k^{*} \mathrm{x}_{\mathrm{o}}, y_{0}^{\prime}=k^{*} y_{o}, \alpha^{\prime}=\alpha / \mathrm{k}, \mathrm{v}_{\mathrm{o}}^{\prime}=k^{*} \mathrm{v}_{\mathrm{o}}$, 
the angular measurements as described by Eq.(2) remain unchanged. However, although there are infinite many number of solutions, but any one of the solutions will give the same least squares estimates of the AZEL state vector. Thus for angular observations taken at time $t=t_{k}$, we obtain:

where

$$
\begin{aligned}
\delta \theta_{k} & =\frac{\partial h\left(\hat{\mathbf{x}}_{k}\right)}{\partial \mathbf{x}_{k}} \delta \mathbf{x}_{k} \\
& =H\left(\hat{\mathbf{x}}_{k}\right) \delta \mathbf{x}_{k} .
\end{aligned}
$$

$$
\frac{\partial h\left(\hat{\mathbf{x}}_{k}\right)}{\partial \mathbf{x}_{k}}=\left[\begin{array}{llll}
\frac{\partial h}{\partial y_{0}} & \frac{\partial h}{\partial \varphi} & \frac{\partial h}{\partial a} & \frac{\partial h}{\partial \nu_{0}}
\end{array}\right],
$$

is a 4 element row vector. Mathematical expressions of each derivative in Eq.(8b) is given in Appendix B. Thus an iterative least squares solution is given by:

$$
\hat{\mathbf{x}}_{\mathrm{k}+1}=\hat{\mathbf{x}}_{\mathrm{k}}+\left(H\left(\hat{\mathbf{x}}_{\mathrm{k}}\right)^{T} H\left(\hat{\mathbf{x}}_{\mathrm{k}}\right)+\lambda I\right)^{-1} H\left(\hat{\mathbf{x}}_{\mathrm{k}}\right)^{T}\left(\mathbf{Z}_{N}-\hat{\mathbf{Z}}_{N}\right)
$$

where $\lambda$ is the numerical convergence control parameter, and I is the identity matrix.

\section{Extension To Out of Plane Trajectory}

Our formulation based on Figure 1 was for a planar case resulting in a purely horizontal bullet trajectory on the FPA. In general the plane formed by the muzzle flash, bullet, and the camera location does not lies on the camera X-Y plane. It will result in a FPA trajectory with a non-zero slope. This problem however, can be handed by a pure rotation of the camera about the $Y$ axis. As shown in Figure 2, a rotation of $\theta_{e}$ radians about the $Y$ axis will put the plane FOB on the $X-Y$ plane. The new angular measurement along the line $O B$ is given by:

$$
\theta_{k}=\sqrt{\theta_{a}^{2}\left(t_{k}\right)+\theta_{e}^{2}\left(t_{k}\right)}
$$

where $\theta \mathrm{a}$ and $\theta \mathrm{e}$ are the azimuth and elevation angles on the FPA. Note also from purely geometric relations, $\theta_{\mathrm{e}}$, bullet elevation angle on FPA, is related to $\varphi_{\mathrm{e}}$, bullet actual shot direction in elevation, by:

$$
\sin \varphi_{e}=\sin \varphi_{a}^{*} \sin \theta_{e}
$$

where again, $\varphi_{a}^{*}$ is the estimated shot direction ( $\angle \mathrm{BFO}$ ) in azimuth after FPA rotation. 


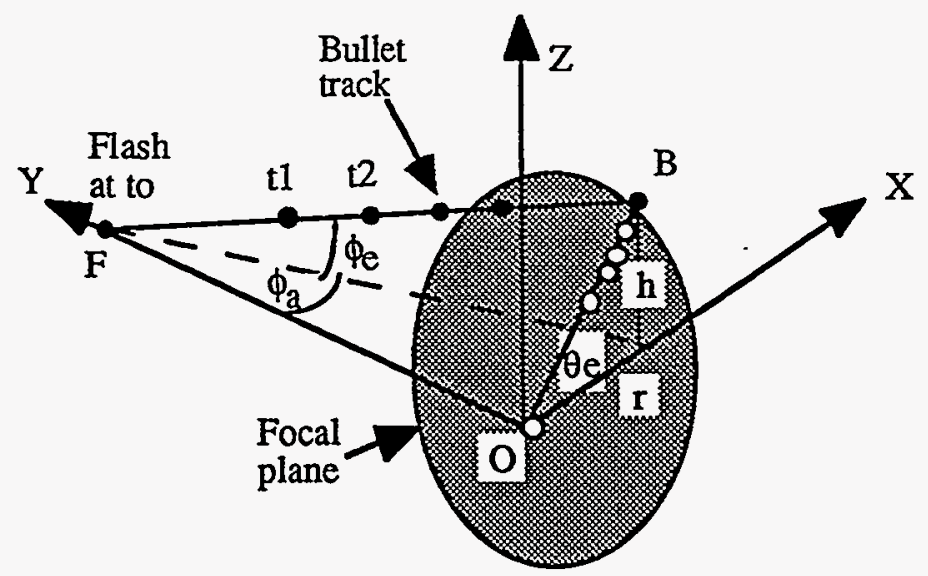

Figure 2 FPA projection of an out of plane trajecotry

\section{Extension to No Muzzle Flash Detection}

For the case where no muzzle flash was detected, we can center the Y-axis (see Figure 1) on the first bullet. Since $t_{1}$ is known, we need to estimate $t_{0}$ or equivalently we can estimate the delay $D=t_{1}-t_{0}$. Letting $D_{k}=t_{k}-t_{1} ; k=1,2,3, \ldots, N$, be the known time differences, then using the relation:

$$
t_{k}-t_{o}=D_{k}+t_{1}-t_{o}=D_{k}+D
$$

Eq.(3) can be rewritten as:

$$
s\left(t_{K}\right)=\frac{1}{\alpha} \ln \left[1+\alpha \mathrm{v}_{0}\left(D_{k}+D\right)\right] .
$$

Eq.(8b) can be extended to include the partial derivative of $h$ w.r.t. $D$ as given by:

$$
\frac{\partial h}{\partial D}=\left[\frac{x_{k} \cos \varphi+y_{k} \sin \varphi}{r_{k}^{2}}\right]\left[\frac{\mathrm{v}_{o}}{1+\alpha \mathrm{v}_{o}\left(D_{k}+D\right)}\right] .
$$

\section{Simulation Studies}

To verify our approach, we developed a simulation capability to evaluate the performance of AZEL track estimation. Figure 3 shows a block diagram of the simulator. The simulator takes inputs of specifications on : weapon types such as muzzle velocity, ballistic coefficient; geometry of gun and camera positions and orientations; and camera parameters such as frame rate, field of view (FOV), pixel size, and angular noise. These inputs along with camera position and orientation parameters allow for the computation of the bullet ballistic state vector. One can also compute the true AZEL state vector on a focal plane array. Noisy measurements of AZEL can then be generated as input for the track estimator. The track estimator generates first the AZEL track and then the ballistic track depending on the amount of apriori information assumed. 


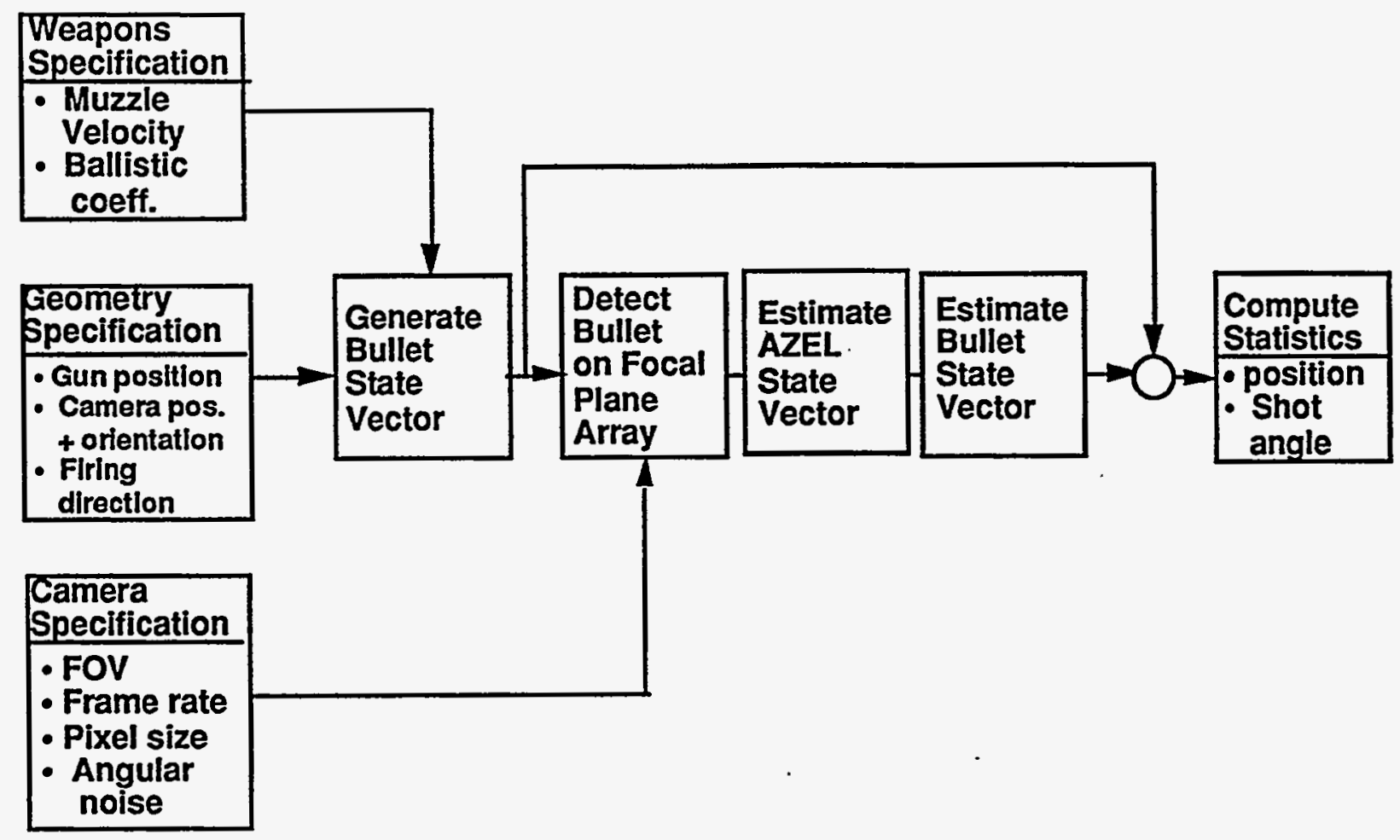

Figure 3 Bullet trajectory estimation simulation block diagram

\section{AZEL Track Estimation with Muzzle Flash Detection}

As stated earlier, angles-only bullet track estimation does not have full observability. However, for AZEL track estimation, it is found that formulation using Eq.(7) has superior angle tracking capability because it is based on a physical model. When a muzzle flash is observed so the time of fire is known, fewer than $25 \%$ of overall bullet observations is needed to reconstruct the whole trajectory. Accurate azimuthal angle tracking in time also allows for precision estimation of the elevation angles using a linear least squares fit between measured elevation and azimuth locations of bullets on the FPA.

Figure 4 shows a simulation of a 0.50 caliber weapon at a distance of 220 meters with a muzzle velocity of $900 \mathrm{~m} / \mathrm{s}$ and a ballistic drag coefficient of about $0.0014 \mathrm{~m}^{-1}$. The weapon is pointing at an angle $68 \mathrm{mrad}$ (4 degrees) to the left of line-of-sight (LOS). Assuming a frame rate of $200 \mathrm{f} / \mathrm{s}$, an ifov of $2 \mathrm{mrad}$, every bullet is detected, and initializing all unknown parameters at least $30 \%$ off (greater than) their nominal values, we notice in Figure 4 that although the distance-to-weapon does not converge (because of the lack of observability), the least squares fit on the azimuthal angle track looks good. The measured angles lie within 1 pixel, $3-\sigma$, while the estimated positions lie within 0.1 pixels of the true track except for the last 10 observations (when the bullet has very high angular velocity on the FPA). The slope of the elevation versus azimuth angles on the focal plane array plot is determined by the weapon's shot direction in azimuth and elevation via Eq.(11). Note also that the error plots in Figure 4 were obtained by subtracting the true value from its estimate. Thus the error goes to zero as the estimate approaches the true. The least squares fit for the last 10 measurements were relatively poor as can be seen in the expanded angular track plot shown in Figure 5. The early portion of the track 
is more or less linear while increasing rapidly near the end. The fit near the end is not as good because it requires in essence a higher order fit which is known to be more noisy [4].
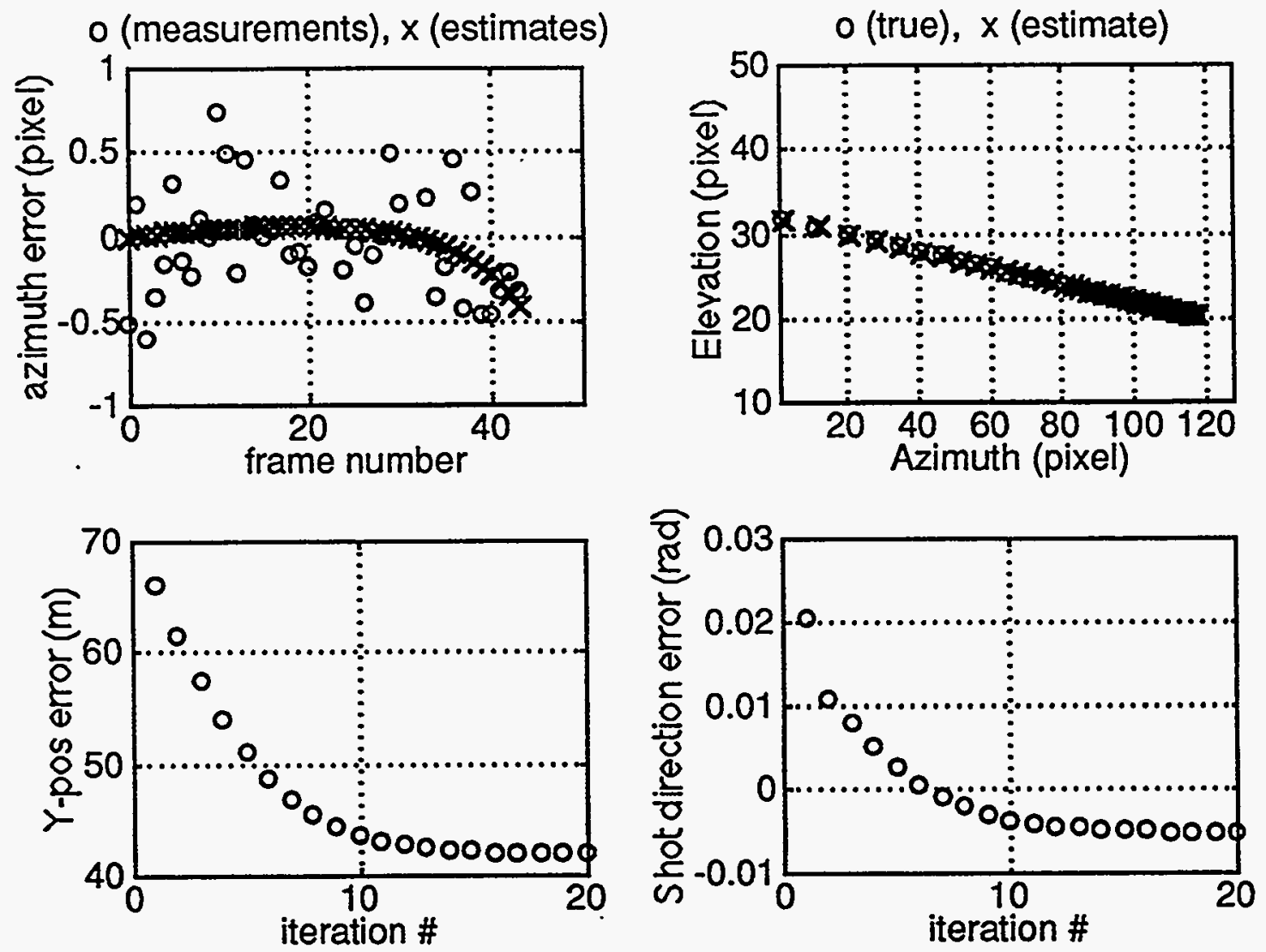

Figure 4 AZEL track simulation with ballistic coefficients and muzzle velocity deviated $30 \%$ from their true state and assuming a bullet is detected at every expected frame.

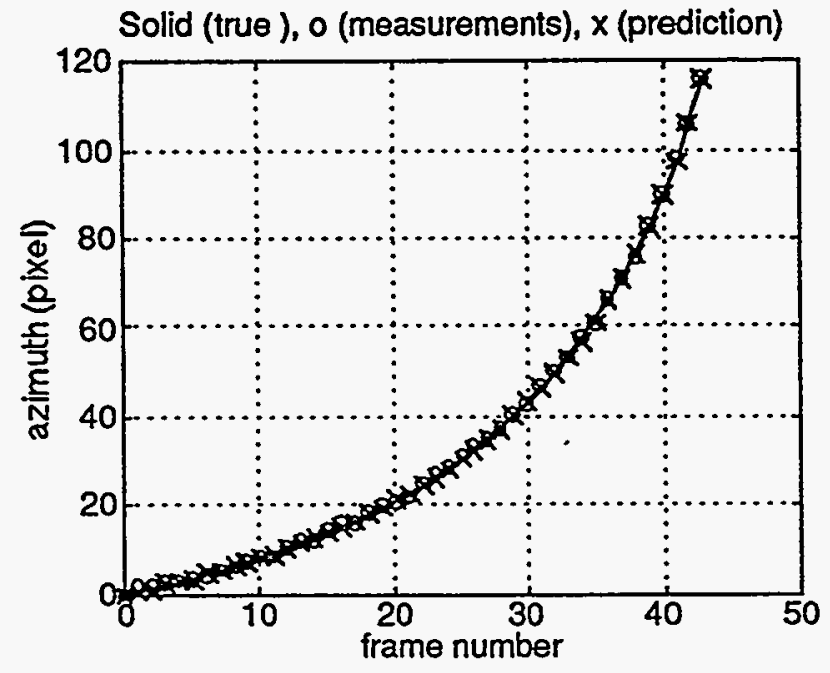

Figure 5 Angular track with bullets detected in every expected frame

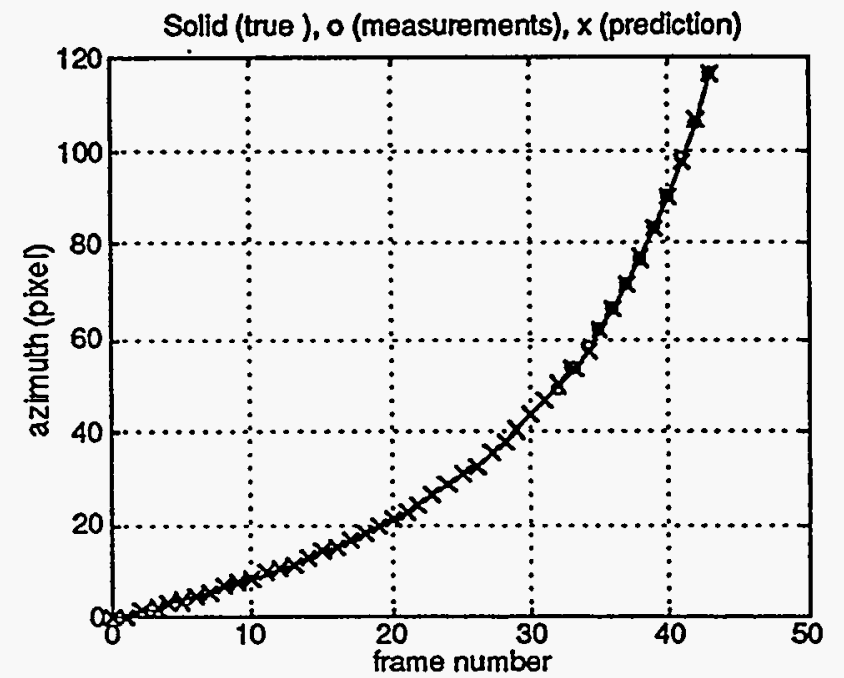

Figure 6 Angular track with only $25 \%$ of bullets detected plus a muzzle flash 
Figure 6 shows the simulation results for the same geometry, but with the bullets detected only in the last $25 \%$ of the frames (i.e., 11 bullets plus the muzzle flash out of an expected total of 43 frames). The corresponding error plots are shown in Figure 7. Note again, neither the range (Ypos error) nor the shot-angle converge, although the AZEL track is excellent - the track is within 0.1 pixels except for the last 5 observations. Also note that the missing $75 \%$ of the bullets were accurately reproduced by the AZEL track estimator.
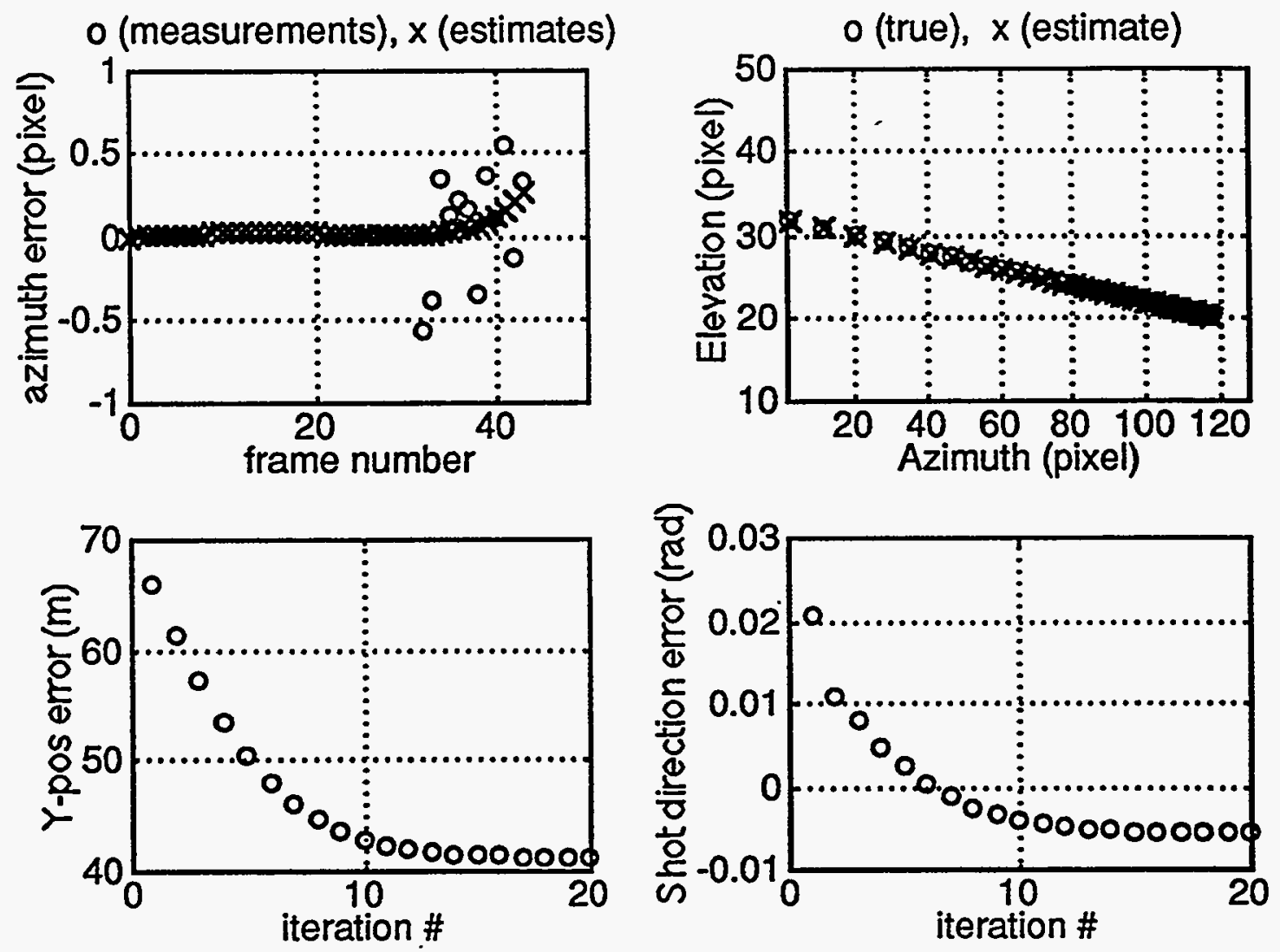

Figure 7 AZEL track simulation with ballistic coefficients and muzzle velocity deviated $30 \%$ from their true state and assuming bullets only detected on last $25 \%$ of the frames.

\section{Metric for Effective Track Error}

We are interested in developing a metric to measure the effective angular displacement of the estimated track and the true track on the FPA. In general the two tracks may be in parallel, crossing, or diverging from each other. We define the effective track error (or displacement) between two tracks on a FPA as the total area subtended by the two tracks divided by the effective pixel length of the true track. Thus let Le and Lt be the estimated and the true tracks on a FPA, then the effective track error is given by:

$$
E T R=\frac{1}{L_{t}} \int_{0}^{L_{t}} \mid L_{e}(\text { pixel })-L_{t}(\text { pixel }) \mid \mathrm{d}(\text { Pixel })
$$


Table 1 shows the track error for three simple geometries. Thus we see that ETR gives an indication of the effective displacement between two tracks. The effective displacement is identical to the actual displacement for the case of two parallel tracks. We are interested in applying this metric of track error to estimate the accuracy of track estimate for different system design parameters such as: frame rates, angular noise, weapon range, and shot-angle.

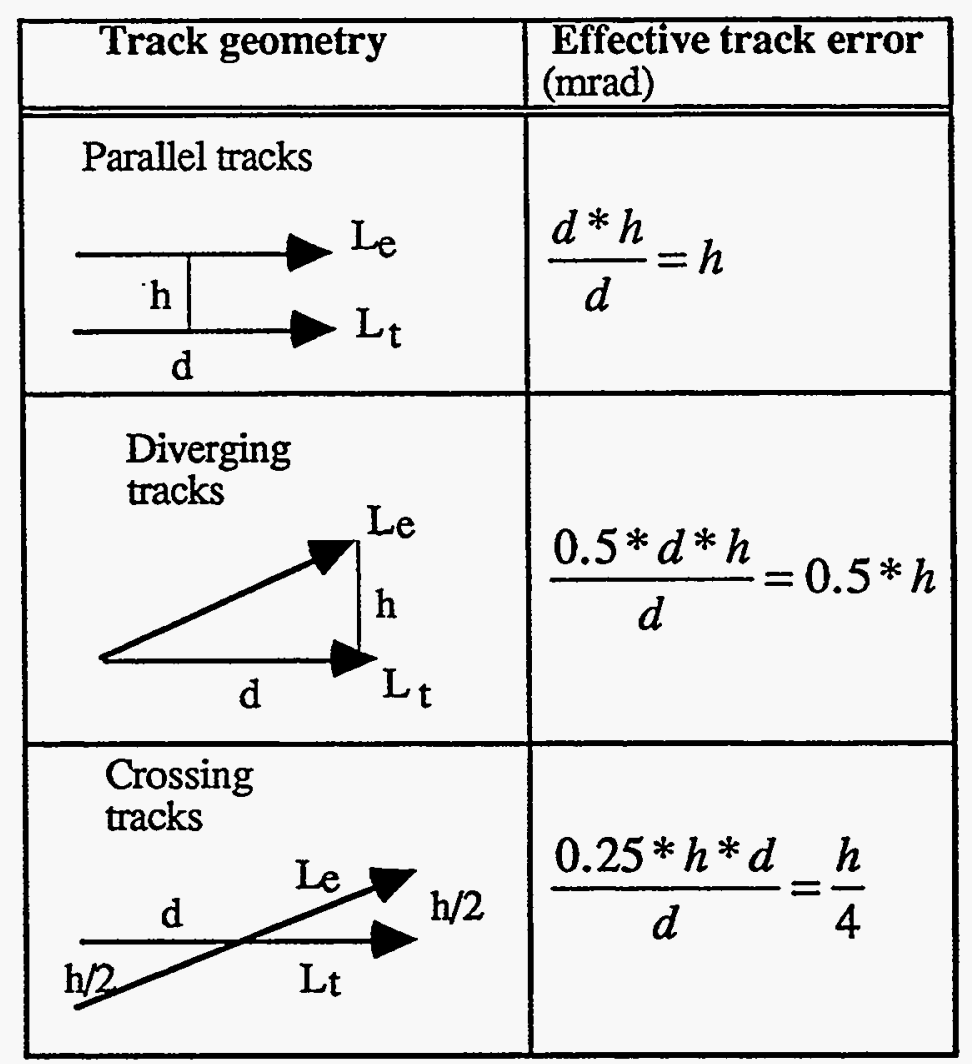

Table 1 Metric for effective error between two tracks

\section{Parametric Sensitivity Study of Track Errors}

Using the simulator we conducted a Monte-Carlo study of angular track accuracy. First we examined the track error versus single-look pixel noise. We assumed pixel noise values of 0.1 to 1 pixel at 0.1 pixel step increments. Using the same geometry and weapon type (i.e., 0.50 caliber machine gun at 220 meters) as discussed earlier, we conducted 30 runs for each noise value. Figure 8 shows the resulting statistical distributions and Figure 9 shows the corresponding mean and sigma value of the same runs. Note that the standard deviation of track error increases as the input pixel noise values increases. The estimate of the mean at each pixel noise level should be accurate to $1 / \sqrt{30}$ of the corresponding standard deviation value. A least mean squares fit of the mean value data for the $200 \mathrm{~Hz}$ frame rate is shown in Figure 10. The result shows that a ten fold increase in noise ( 0.1 to 1$)$ produces a ten fold increase in effective track error ( $50 \mu \mathrm{rad}$ to 500 $\mu \mathrm{rad})$. For faster frame rates, track errors are expected to reduce further since higher frame rates produce more observations for a given observation period. Figure 11 summarizes the result for 
frame rates of $200 \mathrm{~Hz}$ to $500 \mathrm{~Hz}$. [The crossing of the $300 \mathrm{~Hz}$ and $400 \mathrm{~Hz}$ lines is due to the statistical uncertainty in the mean estimates.] Note that there are no appreciable error differences between $400 \mathrm{~Hz}$ and $500 \mathrm{~Hz}$.

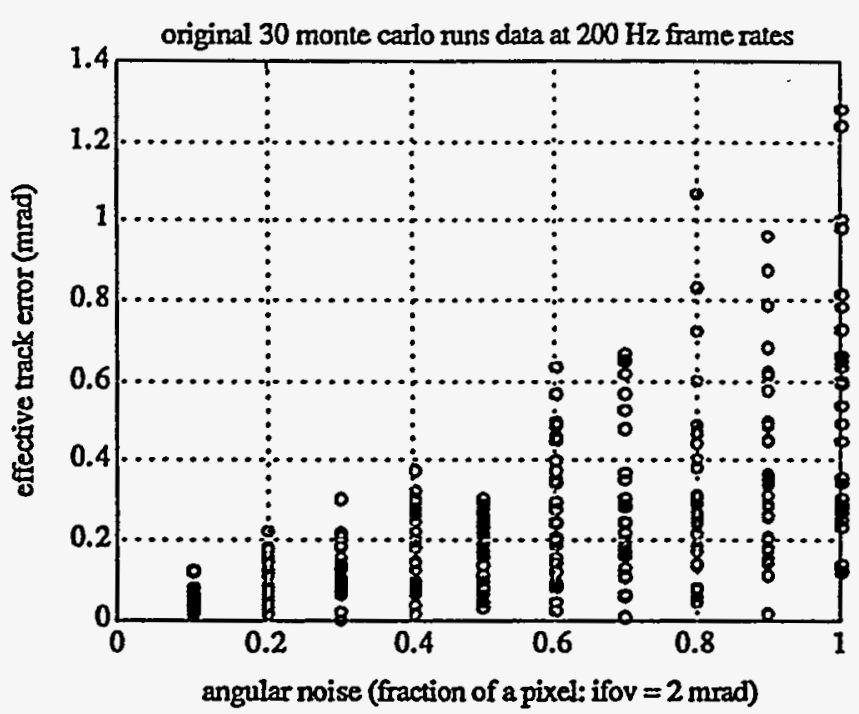

Figure $8 \quad 30$ Monte-Carlo runs at each pixel noise and at 200 frame rate

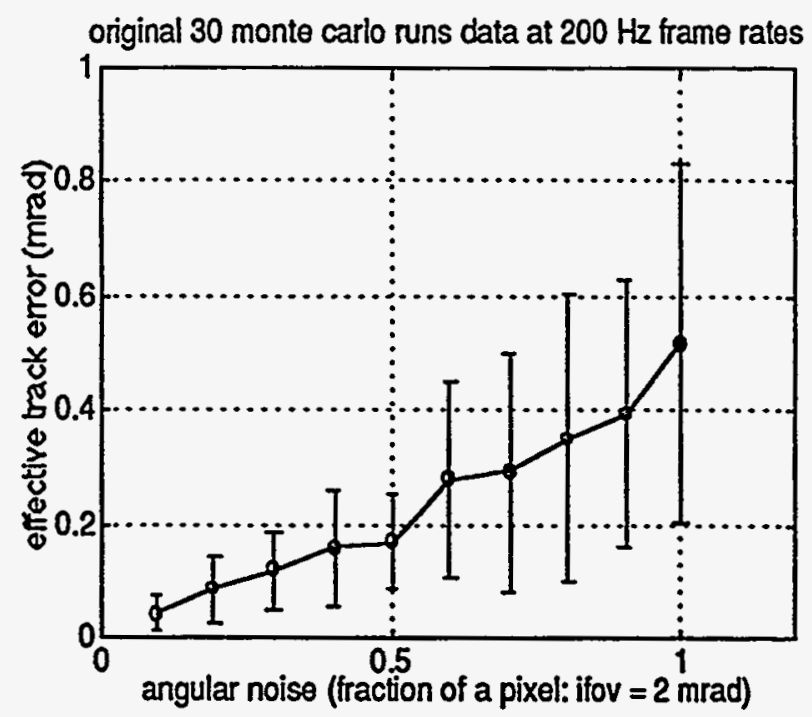

Figure 9 Standard deviations of 30 Monte-Carlo runs at each angular noise

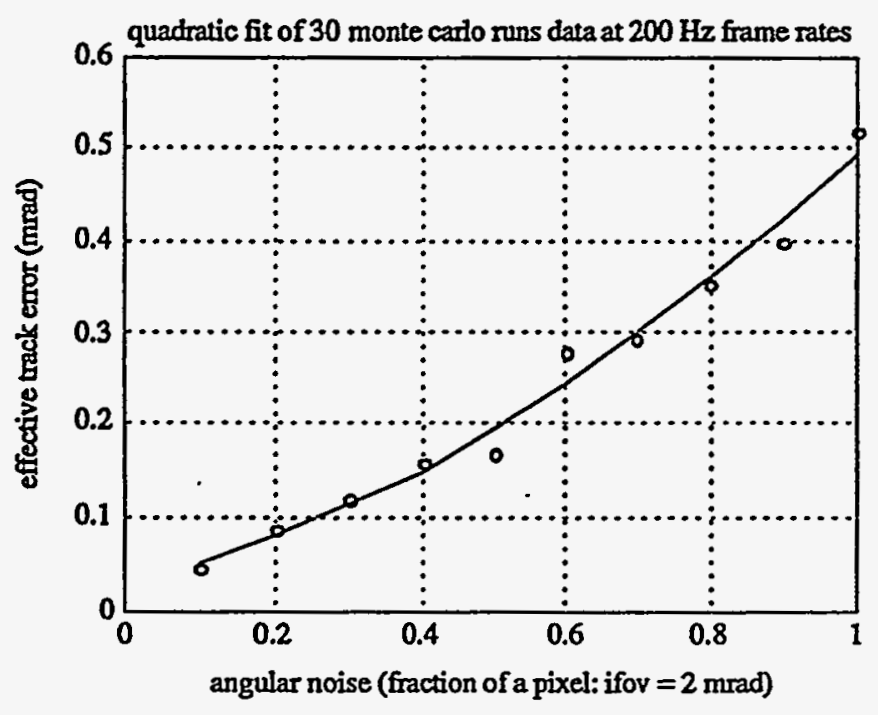

Figure 10 Least squares fit of the mean values

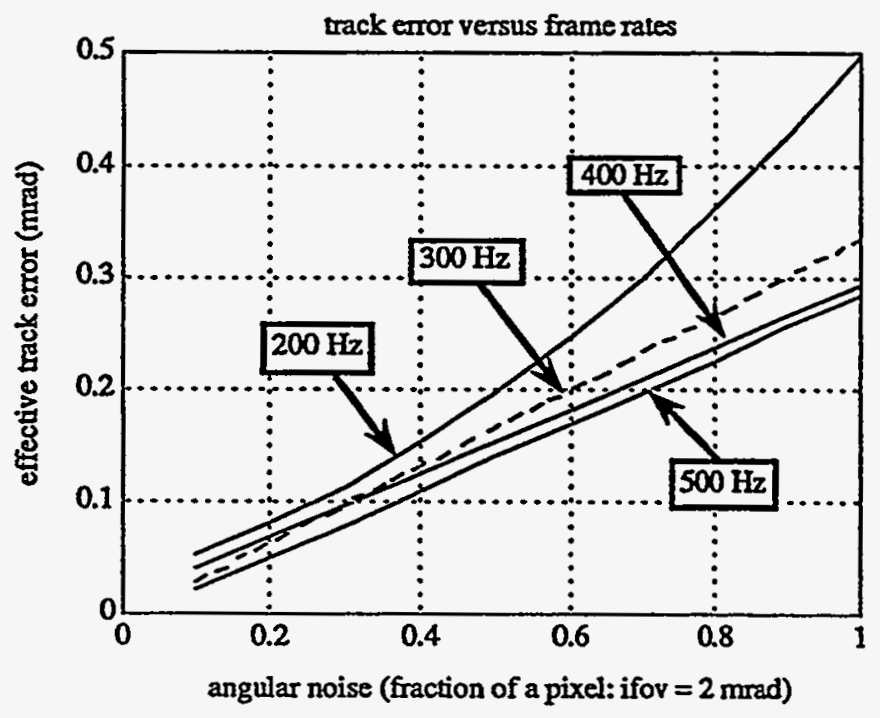

Figure 11 Track error versus frame rates

We also investigated the track errors at three different ranges $(100 \mathrm{~m}, 200 \mathrm{~m}, \& 300 \mathrm{~m})$ and for two different field-of-view sensors $(128 \times 128$ FPA-15 degrees \& $512 \times 512$ FPA-60 degrees). For each condition, we evaluated the track error for shot-angles ranging from 2 degrees to 80 degrees. For all the runs a 0.5 pixel noise value was assumed. Figures $12 \& 13$ summarize the results. Three observations can be made: (1) for a given shot-angle, track errors increase with 
longer ranges, (2) for the $15^{\circ}$ FOV sensor, track errors increase with larger shot-angles, and (3) for the larger $60^{\circ}$ FOV sensor, track errors decrease with increasing shot-angles. These observations may be explained as follows correspondingly: (1) track errors increase with longer ranges because longer range implies larger pixel noise because of lower signal-to-noise ratio (this effect is built into our model); (2) track errors increase with the narrow FOV for greater shotangles because larger shot-angle means a smaller number of total frames containing a bullets as it moves across the sensor FOV, and a smaller number of observations implies greater track uncertainty; and (3) for the larger FOV sensor, track errors decrease with increasing shot-angles because of two favorable conditions: larger shot-angle means more detected bullets in the FOV, and the angular track as a function of time becomes more linear and can be fit to a lower-order curve. A lower order least squares fit also increases the noise rejection capability because of its smaller equivalent bandwidth [4].

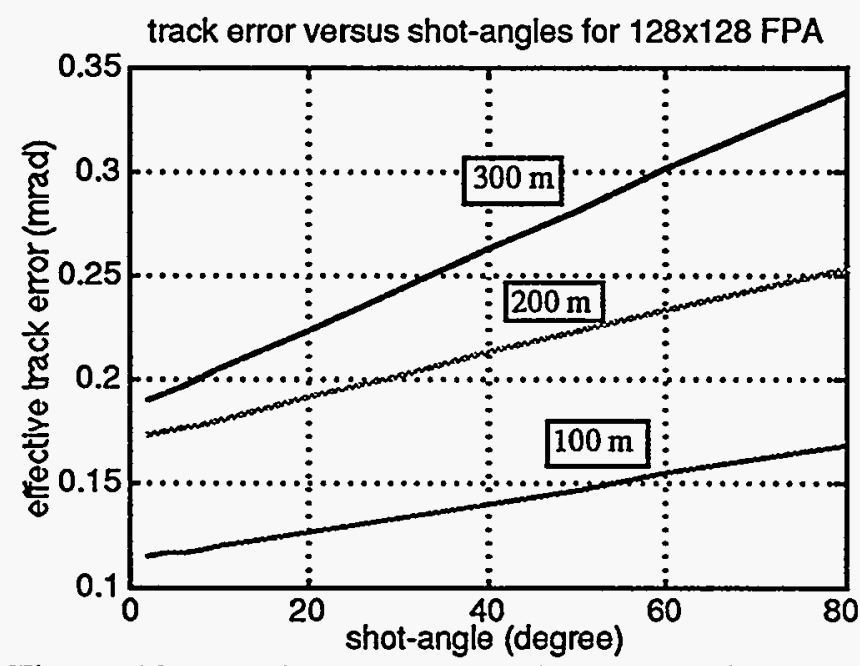

Figure 12 Track error versus shot-angles for a $128 \times 128 \mathrm{FPA}$ at different ranges

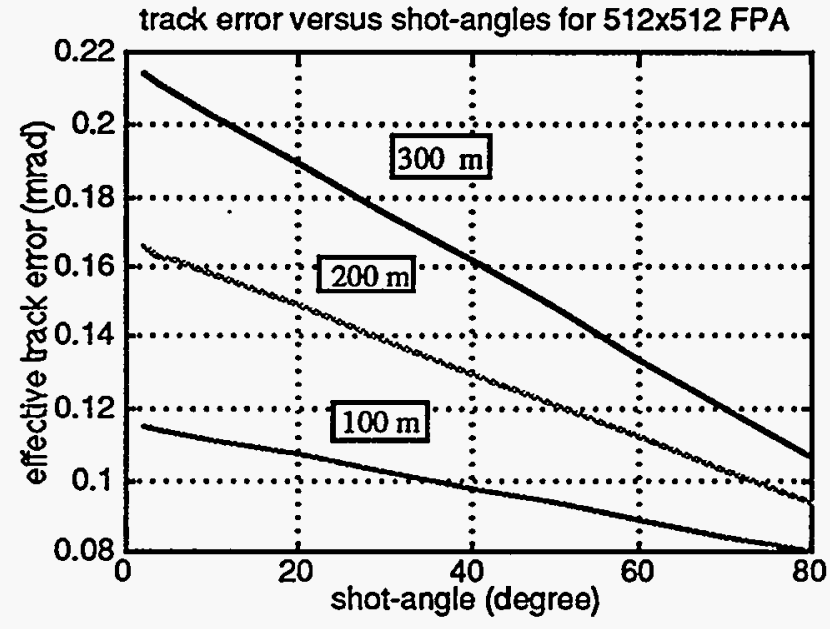

Figure 13 Track error versus shot-angles for a $512 \times 512$ FPA at different ranges

\section{AZEL Track Estimation with No Muzzle Flash Detection}

We extended the simulation to study the case where the muzzle flash was not detected either because it was not within the field-of-view of the sensor or it was somehow suppressed. To estimate the location of the muzzle flash, we need to estimate the time delay parameter D as shown in Eq.(12). Since we know $t_{1}$, time of first bullet detection, $t_{0}$, time of muzzle flash is obtained from subtracting $D$ from $t_{1}$. The additional equation needed in the least squares estimation procedure is Eq.(14). The time delay parameter is estimated based on the muzzle velocity and ballistic coefficient. Again we used the same firing geometry and sensor characteristics as in the case under AZEL track with muzzle flash detection discussed earlier. Starting guess of the solution state vector is $30 \%$ greater than the true values. For the following studies, initial guess of the time delay parameter was set at $50 \%$ of it true value.

Figure 14 shows the azimuth track as a function of detection frames with the assumption that bullets were detected only in the last $25 \%$ of the frames. It can be seen that the predicted bullet locations are very close to the true locations in frames where bullets are actually detected, and continue to be predicted very close to the true trajectory even on frames where no bullets are detected. Note the angular error of the gun location is small. The exact value is given in Figure 15. The angular error plot shows that the prediction of the muzzle flash time is about 1.3 frames short, and the gun location error is approximately $3 \mathrm{mrad}$. 


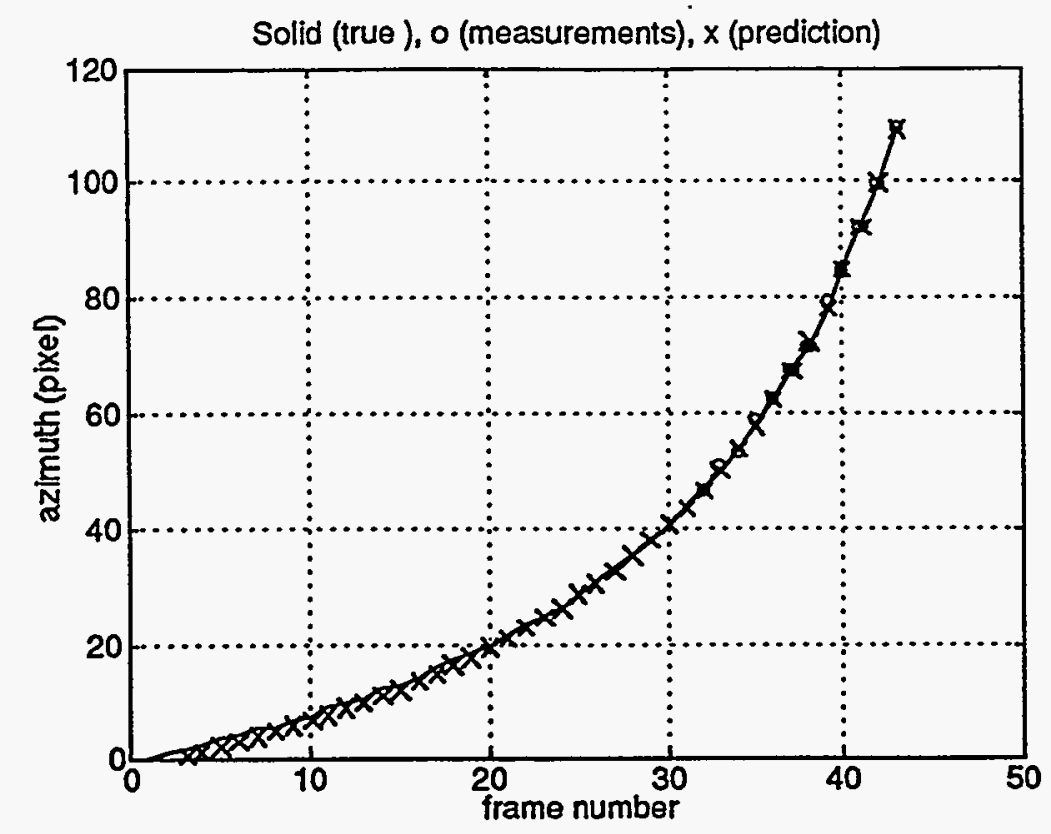

Figure 14 Azimuth tracking with no muzzle flash detection
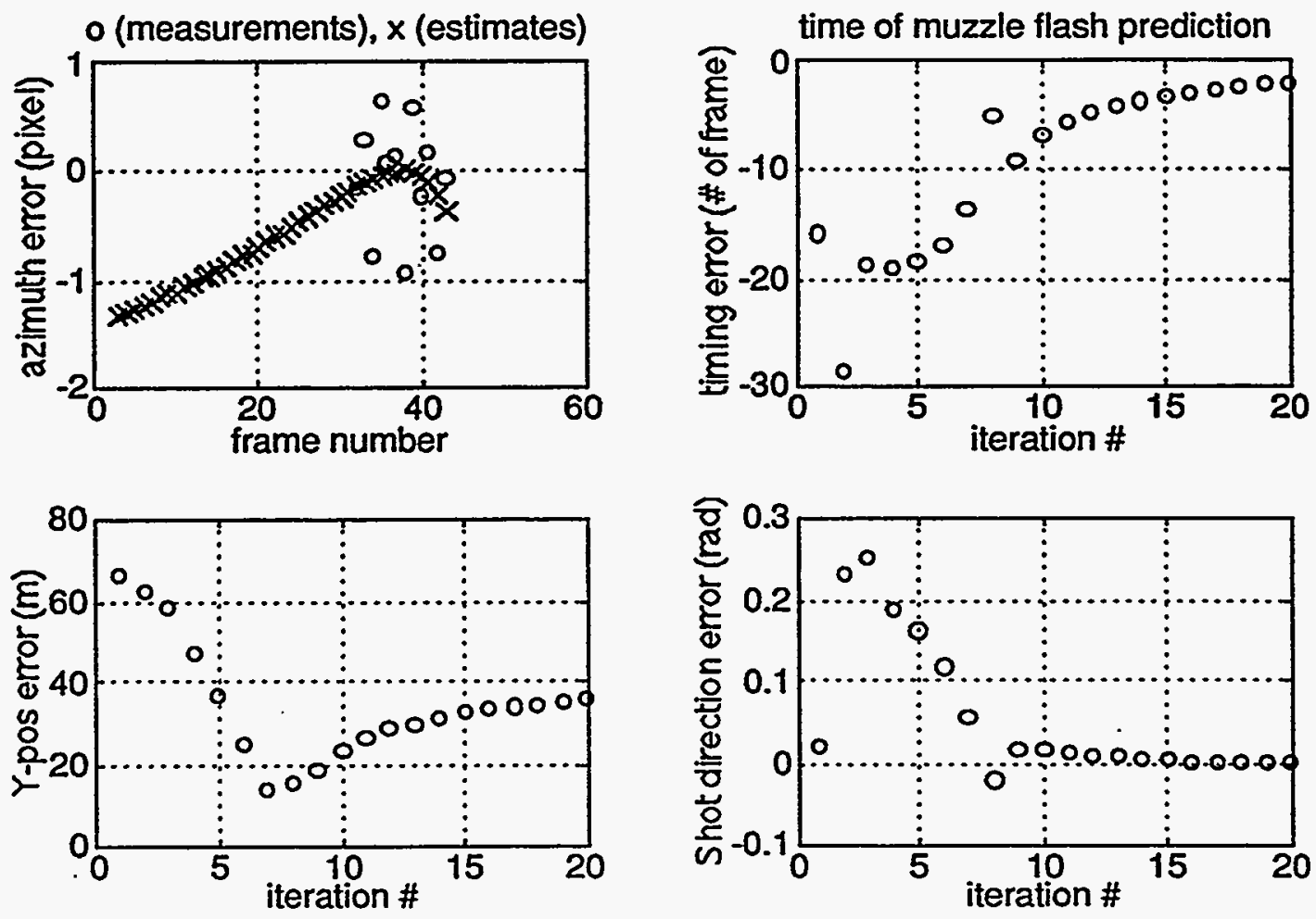

Figure 15 AZEL track with bullets detected in the last $25 \%$ of frames. (no muzzle flash) 

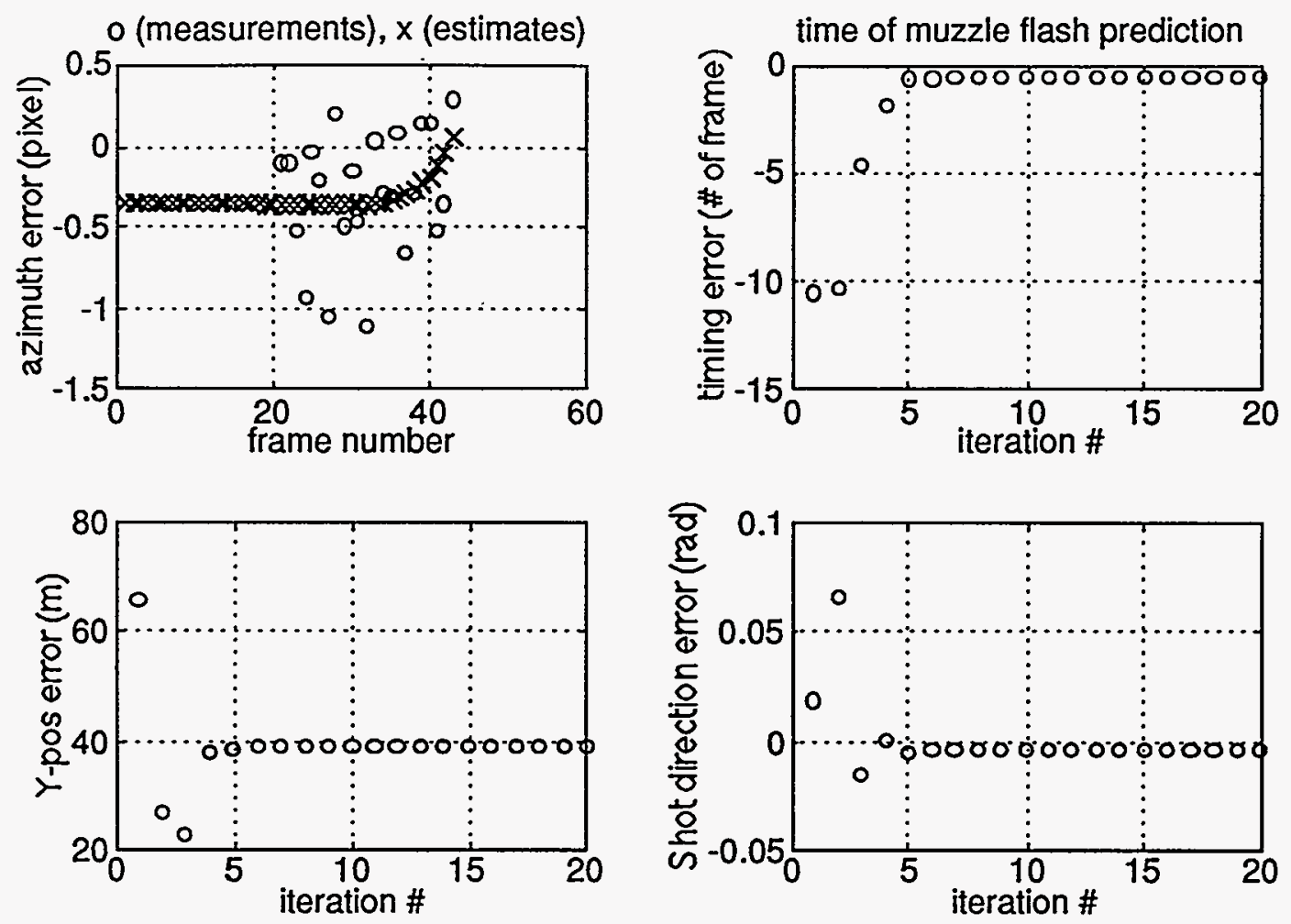

Figure 16 AZEL track with bullets detected in the last 50\% of frames. (no muzzle flash)

Figure 15 also shows that although the range did not converged, but both the muzzle flash timing error and the shot-angle prediction error indicated convergence. Figure 16 confirmed the observation that with more bullets detected, convergence improved. Muzzle flash location error is now less that 0.5 pixels. Both shot-angle and time of muzzle flash essentially converged at a factor of 2 or more faster.

Figure 17 shows improved performance with the assumption of detecting more bullets. Using the assumption that $75 \%$ of the bullets were detected, the muzzle flash location is now less that 0.1 pixels.

Evidently, in many shot geometries the sensitivity of the time delay parameter to muzzle velocity error is less than 1:1. In the case of Figures $15-16$, a $30 \%$ velocity error resulted in a $3 \%$ time delay error, and a correspondingly small error for the source location. 

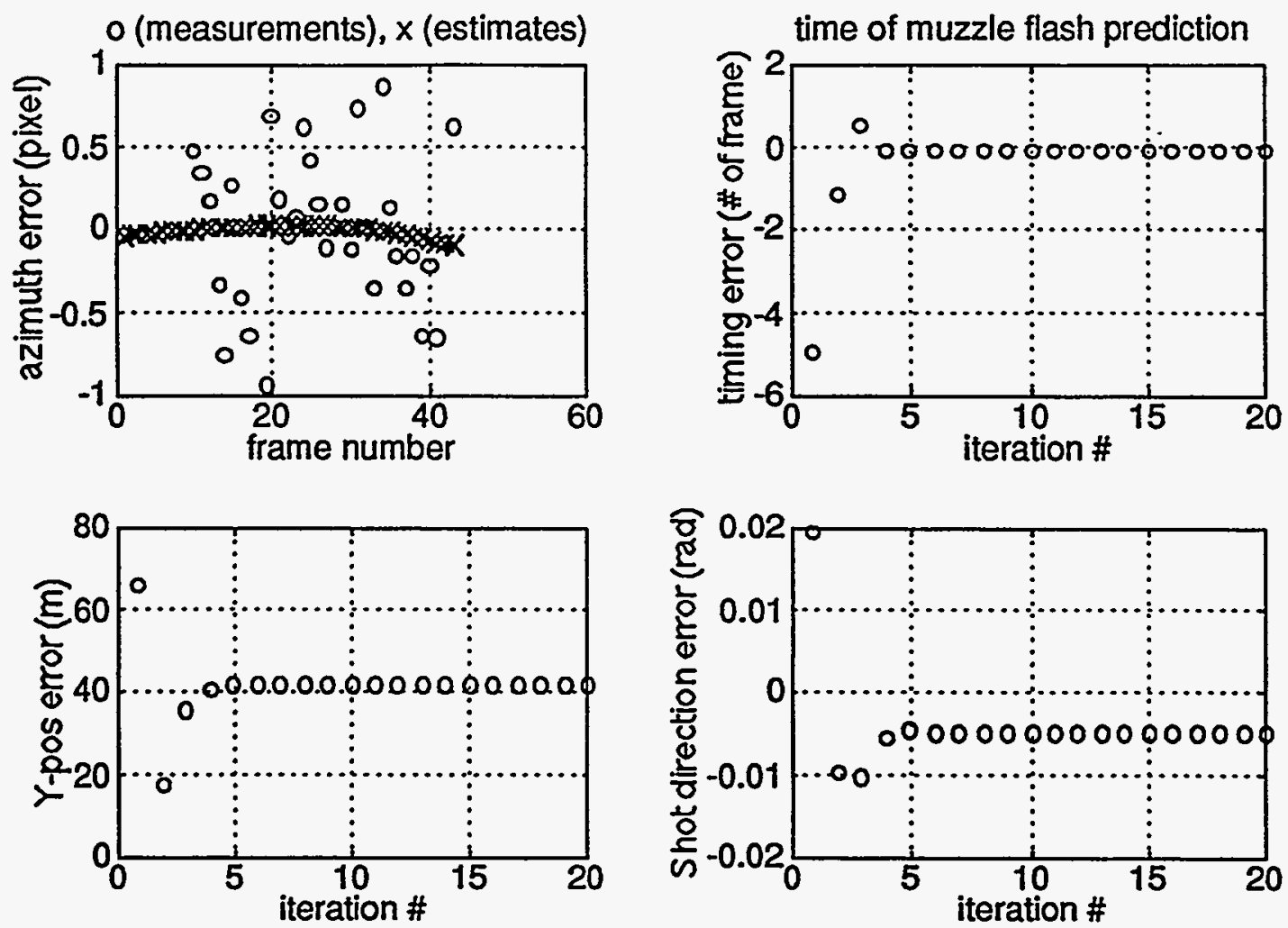

Figure 17 AZEL track with bullets detected in the last 75\% of frames. (no muzzle flash) 


\section{Ballistic State Vector Estimation}

For AZEL state vector estimation, we concluded that given a reasonable set of initial conditions for the ballistic coefficient and muzzle velocity, the AZEL state vector converged nicely although the target range and shot direction did not converge simultaneously. However, we suspect that if we choose the correct values of ballistic coefficient and muzzle velocity, the target range and shot direction will converge to their true values. This is indeed the case as shown in Figures 18 and 19. Note in Figure 18, we assumed the bullet is detected on every frame while in Figure 19 we assumed bullets are detected on only the last $25 \%$ of frames in addition the muzzle flash.

Note also that in Figure 19, although we used only $25 \%$ of the overall measurements, the azimuth angle track error is less than 0.1 pixels over the missing bullet locations.
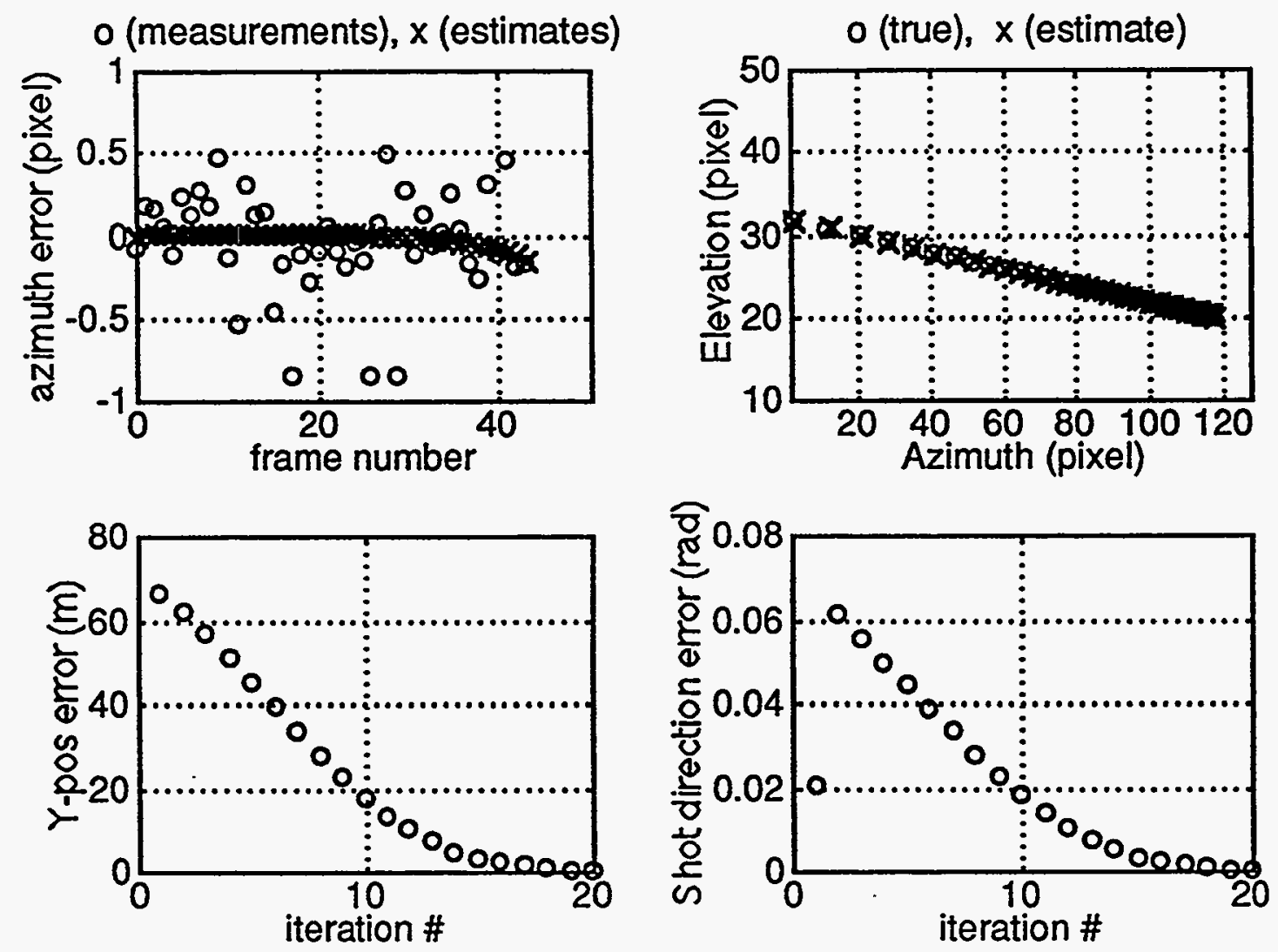

Figure 18 Simulation of bullet track using the exact ballistic coefficient and muzzle velocity. Note that both range and shot direction converged to their true values. 

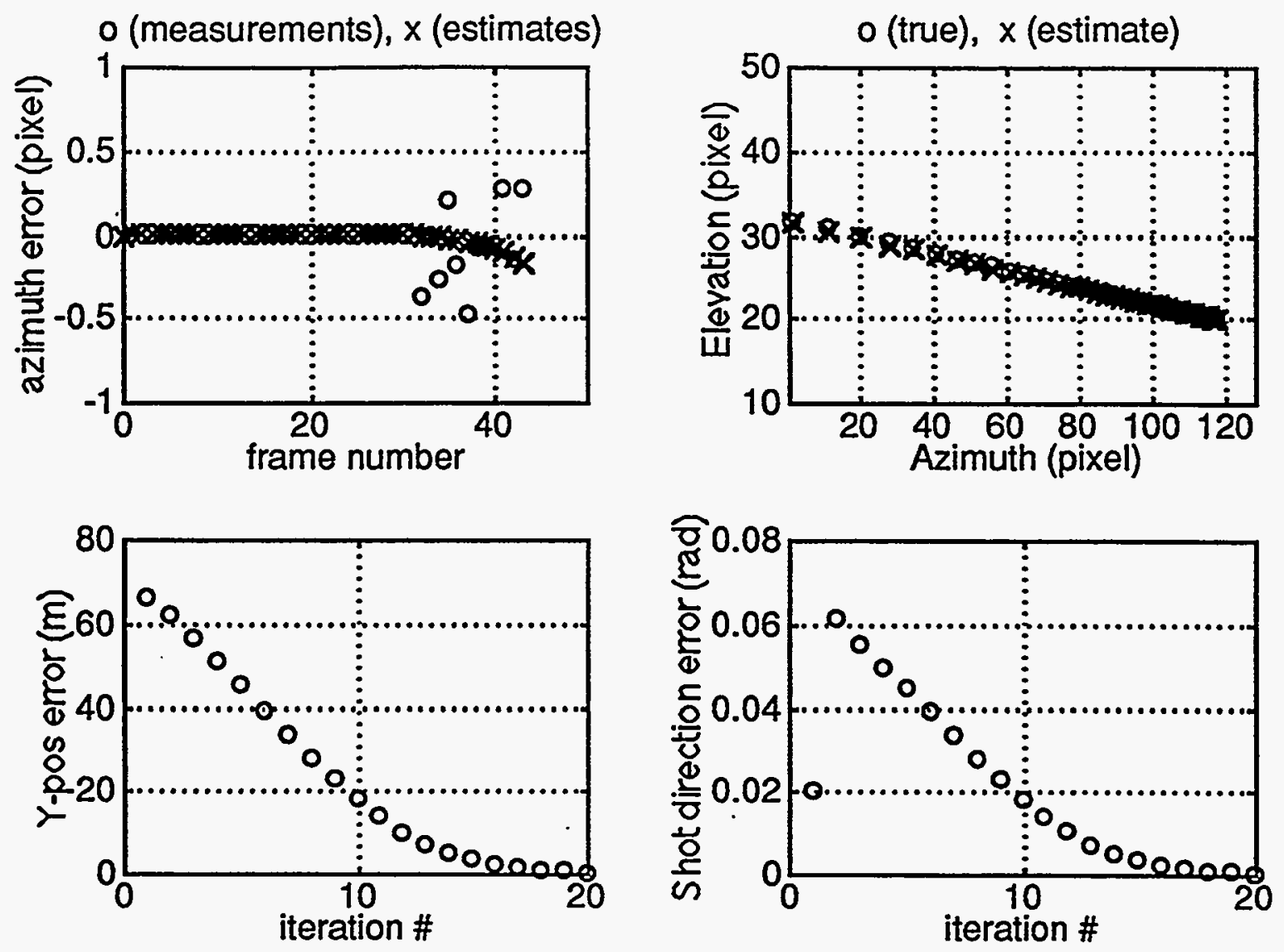

Figure 19 Simulation of bullet track using the exact ballistic coefficient and muzzle velocity. Note that both range and shot direction converged to their true values where we assumed the bullet detected on only the last $25 \%$ of frames.

\section{Error Analysis of Source Location Parameters}

We are interested in the error sensitivity of source location parameters to percentage error in our estimate of ballistic parameters. We have shown that using the exact knowledge of ballistic parameters (muzzle velocity and ballistic coefficient), the ballistic state vector is observable from measured AZEL data. The ballistic coefficient is a function of mass, aerodynamic drag coefficient, and effective surface area. The ballistic coefficient of a weapon can be obtained from a firing table and is only a function of mach number, but the latter is also dependent on the muzzle velocity. Thus knowledge of muzzle velocity is crucial. Even if the weapon type is known, the muzzle velocity may change significantly from its nominal value due to different conditions of the gun barrel and ammunition. In a given experiment the muzzle velocity can be measured accurately. Muzzle velocity may also be available for non-real-time ballistic track reconstruction, for example during analysis of a crime. However, in a realistic realtime environment, it may not be possible to know the muzzle velocity with great accuracy.

Figures 20 and 21 show the sensitivity of range and shot-angle to variation in muzzle velocity. An almost one-to-one percentage error is observed for the range. On the other hand, the shot-angle is non-linearly related to error in muzzle velocity. Note that the error is significantly more sensitive in the negative direction. 
Figures 22 and 23 shows the sensitivity of range and shot-angle to variation in the ballistic coefficient. Both errors are linear and are approximately scaled down by a factor of six in percentage error. For example, a $30 \%$ error in ballistic coefficient produces only a $5 \%$ error in range and shot-angle.

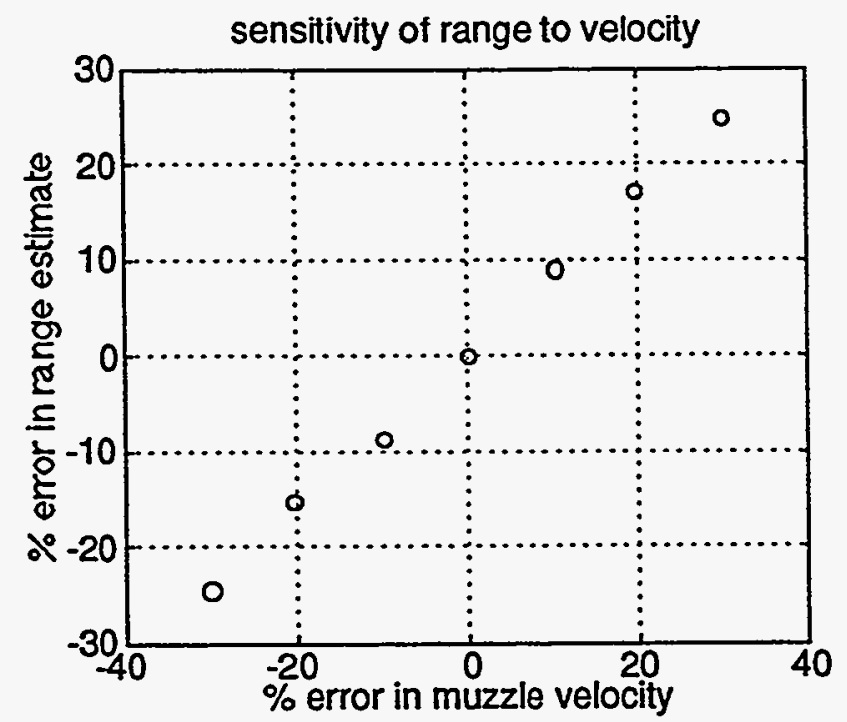

Figure 20 Sensitivity of range to velocity

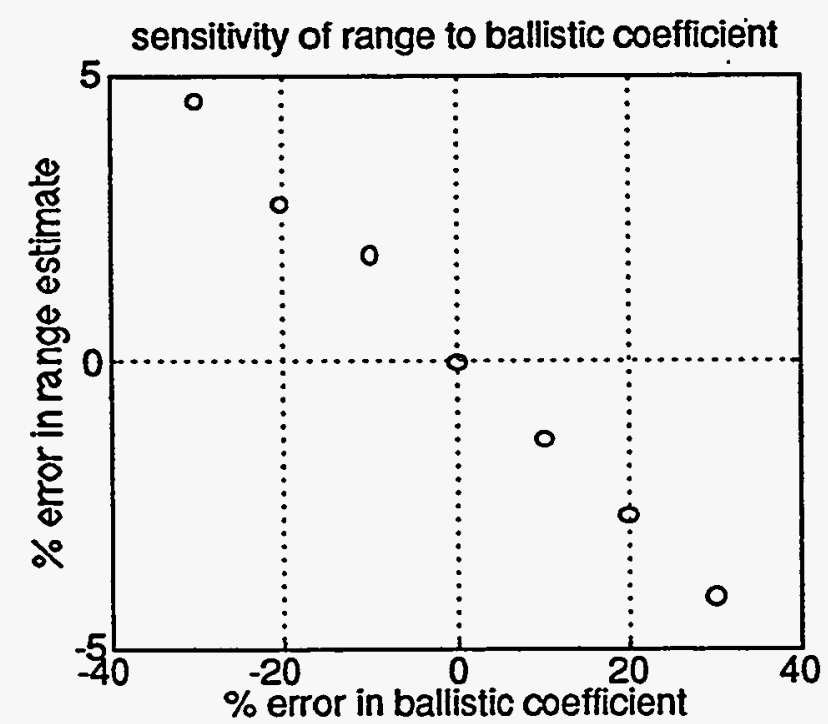

Figure 22 Sensitivity of range to ballistic coefficient

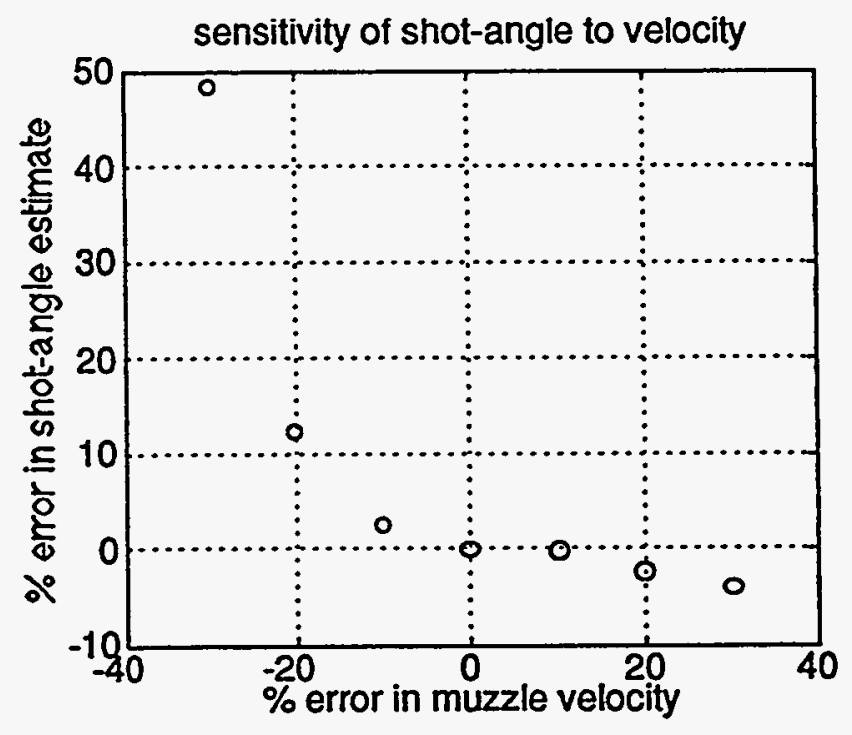

Figure 21 Sensitivity of shot-angle to velocity

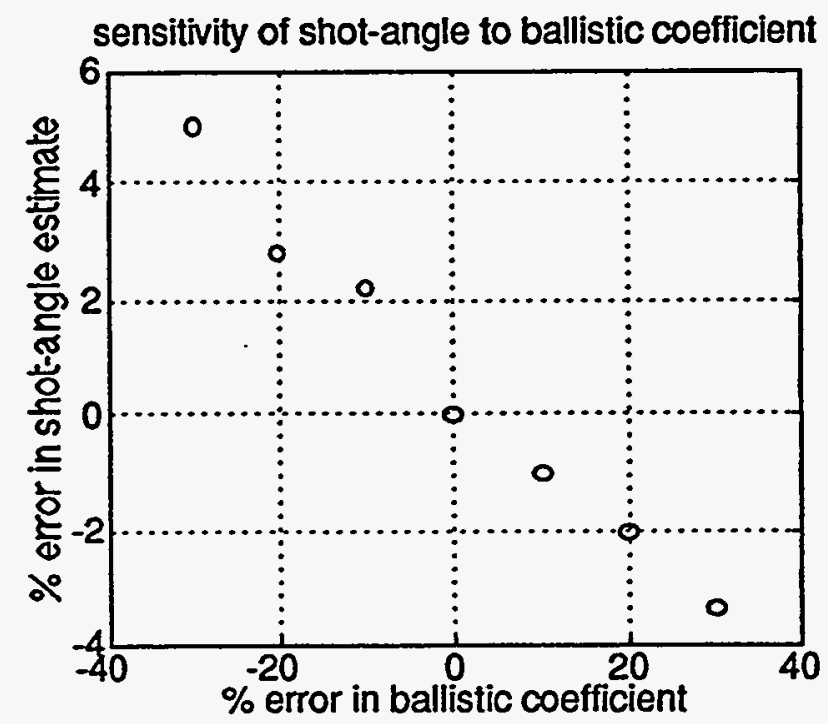

Figure 23 Sensitivity of shot-angle to ballistic coefficient 


\section{Experimental Setup}

We analyze one of the many bullet tracking experiments done at LLNL's Site 300 firing range, and compare the trajectory of a real bullet to our estimated trajectory. Appendix C shows the locations of various weapons, camera and targets. The particular experiment we selected for analysis was conducted in August 1994 with a 0.50 caliber M2HB machine gun. The geometry of the experiment is shown in Figure 24, where the $x, y, z$, locations of the target, camera, and gun were given. The gun was firing to the left of camera-to-gun LOS. Note that in Figure 20 we corrected for the gun muzzle location by adding 1.14 meters in $\mathrm{X}$ and -1.3 meters in $\mathrm{Z}$ to the surveyed point which represents the tripod mount for the gun. Also we corrected for the actual target location by adding $-0.6 \mathrm{~m}$ in $\mathbf{Z}$ to the surveyed location. The geometric parameters such as shot angle and range can be computed as follows:

Let

$$
\begin{aligned}
& \mathbf{A}_{\mathrm{cg}}=\text { distance vector from gun to camera, } \\
& \mathbf{A}_{\mathrm{tg}}=\text { distance vector from gun to target, }
\end{aligned}
$$

then we found that

$$
\text { range }=|\operatorname{Acg}|=220 \text { meters }
$$

and the shot angle is

$$
\begin{aligned}
\varphi_{a} & =\cos ^{-1}\left(\frac{|\mathrm{A} c \mathrm{~g}|^{2}+|\mathrm{A} t \mathrm{~g}|^{2}-|\mathrm{A} t \mathrm{c}|^{2}}{2^{*}|\mathrm{~A} c \mathrm{~g}||\mathrm{A} t \mathrm{~g}|}\right) \\
& =0.064(\mathrm{rad}),
\end{aligned}
$$

Also the 0.50 caliber muzzle velocity was measured by an Oehler chronograph and found to be 900 meters per second with $\pm 1 \%$ variation from shot-to-shot. Also the inverse ballistic coefficient is found to be $5.24 \times 10^{-4} \mathrm{~m}^{-1}$.

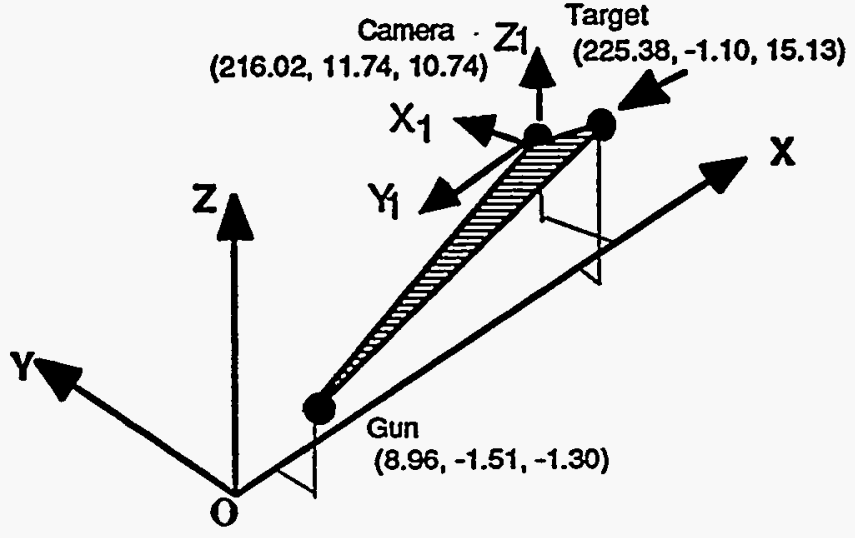

Figure 24 Geometry of bullet tracking experiment

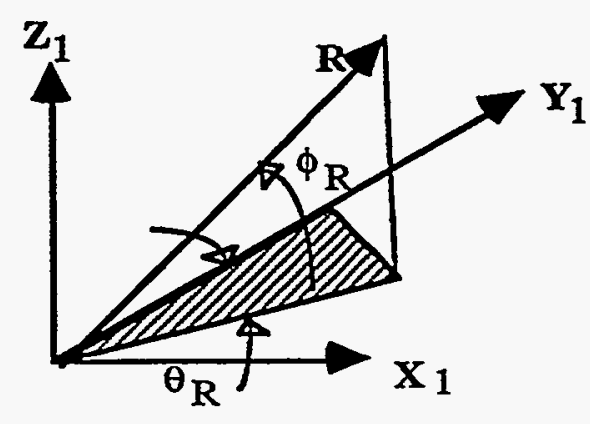

Figure 25 Reference vector in the $X_{1}, Y_{1}, Z_{1}$ coordinate system 


\section{Bullet Trajectory Reconstruction}

We will briefly summarize the exact procedure for trajectory reconstruction. Our problem is the following. Given the surveyed locations of the gun, camera, and target, find the resulting true bullet trajectory on the camera FPA. We will make use the knowledge of muzzle flash location on the camera for this purpose. We noticed that the muzzle flash expanded rapidly to a radius as large as 5 pixels as the high pressure hot gas was pushing the bullet out of the gun barrel. Thus during the expansion cycle, exact location of the flash is difficult to determine. However, after the expansion cycle, the pressure around the muzzle dropped rapidly resulting in a contraction cycle with an accompanying drop in temperature. The contraction cycle actually reduced the radius of the flash to smaller than 1 pixel. Subpixel resolution of the flash location may be feasible if a better model of the muzzle flash dynamics is available.

In order to accurately reconstruct the bullet trajectory on the FPA, we need to accomplish two steps: (1) reconstruct the bullet trajectory in the firing range coordinate (i.e., X,Y, Z); (2) map the trajectory to the camera coordinate frame denoted by the system $\left(\mathbf{X}_{\mathbf{C}}, \mathbf{Y}_{\mathbf{c}}, \mathbf{Z}_{\mathrm{C}}\right)$. The detail of the reconstruction and the coordinate transformation matrix are presented in Appendix D.

\section{Bullet Tracking Experiments and Performance Validation}

Using the geometry and bullet parameters as computed in the last section, we reconstructed the trajectory using Eqs.(19) \& (25). Figure 26 shows the expected angular track as a function of time. Expected measurements were computed at every frame time. Also the AZEL track as appearing on the camera FPA is shown in Figure 27.

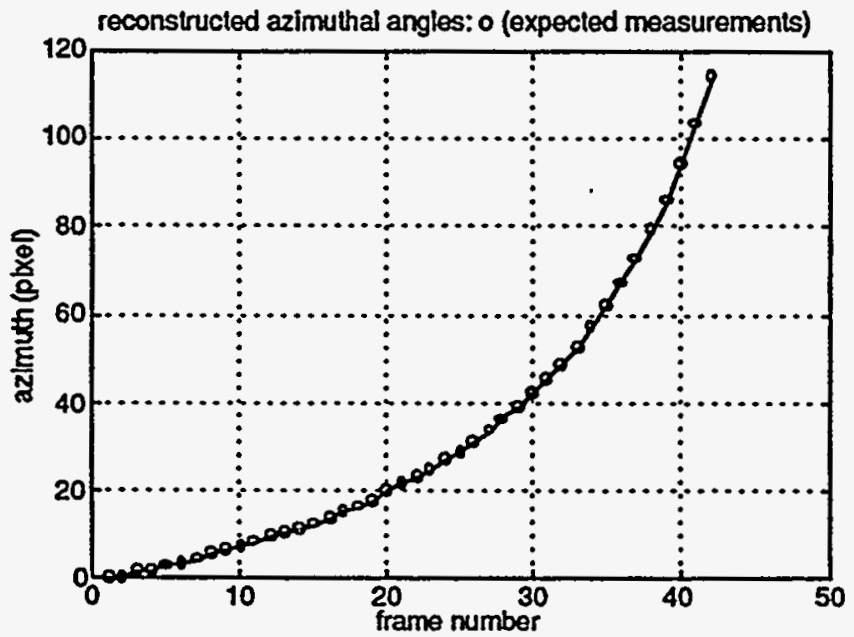

Figure 26 Generation of azimuthal angular track as a function of time using the firing range data.

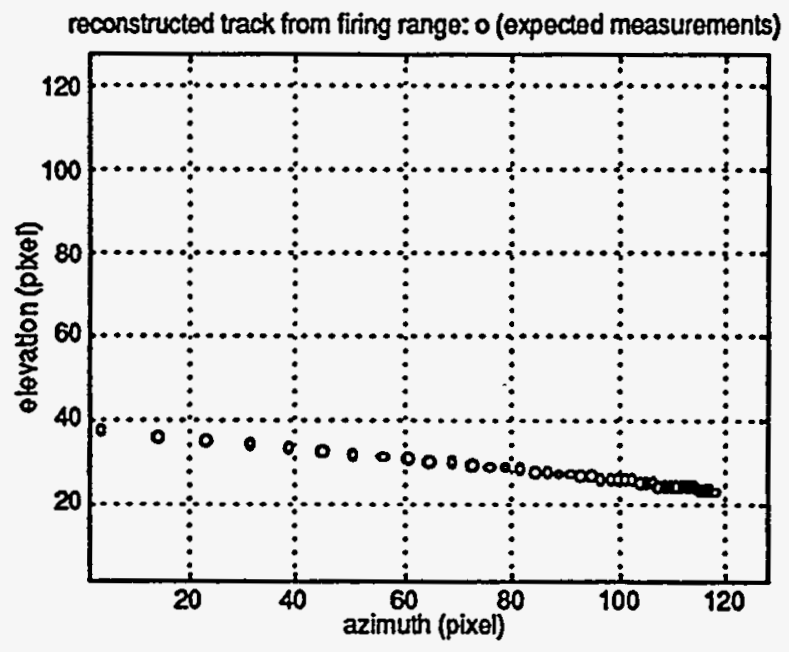

Figure 27 Generation of AZEL track as a function of time using the firing range data.

Figure 28 shows an image of the experimental site. In the background of the bullet track is a steep hill. We analyzed the data on one of the shots known as d40809dat2_0.raw. The weapon is a 0.50 caliber machine gun located approximately at 220 meters from the camera and was shooting at a target $64 \mathrm{mrad}\left(\sim 4^{\circ}\right)$ to the left. Figure 29 shows a composite image from the bullets on 45 frames. Note that the bullets are visible in the processed data on almost every frame back to the muzzle flash of the gun. 


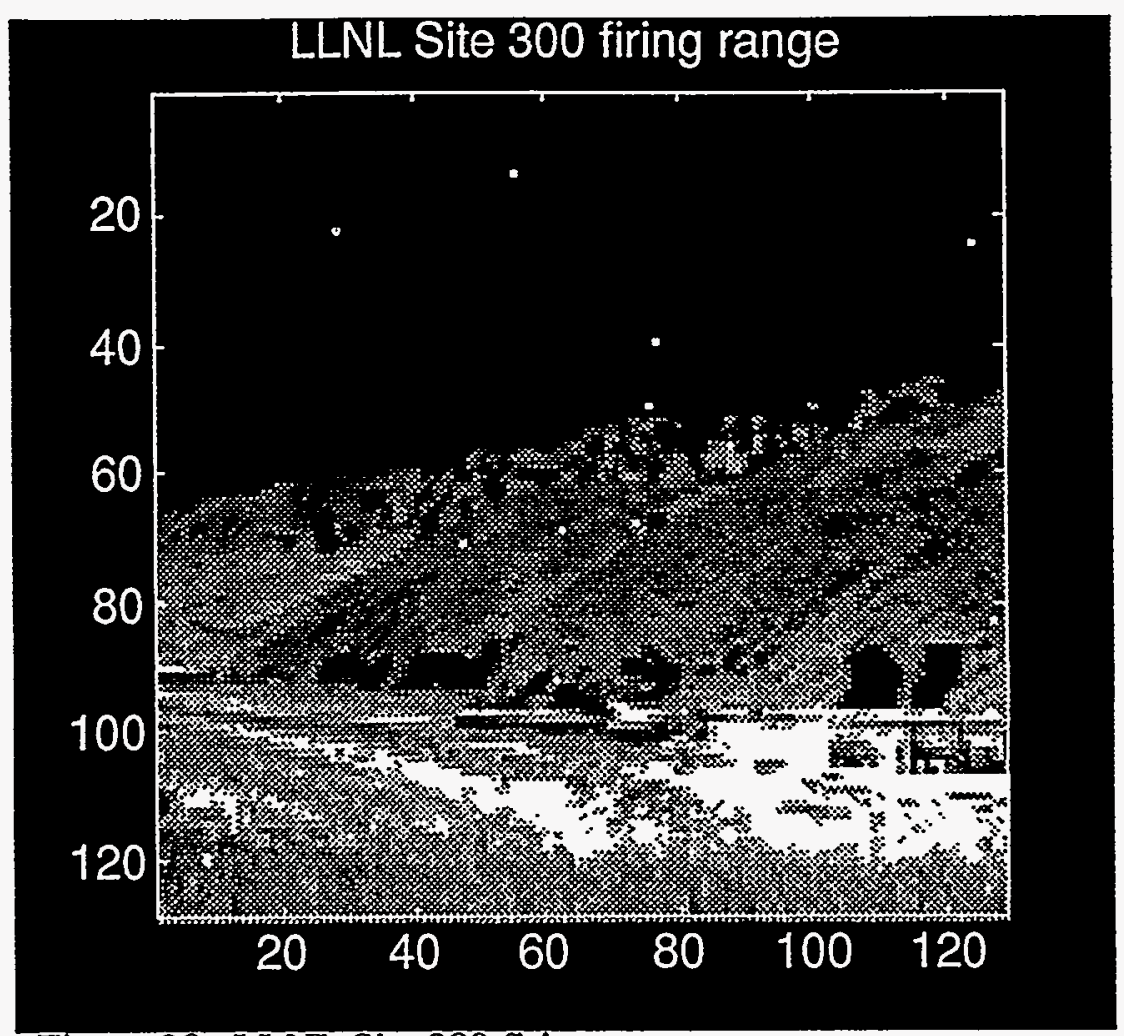

Figure 28 LLNL Site 300 firing range

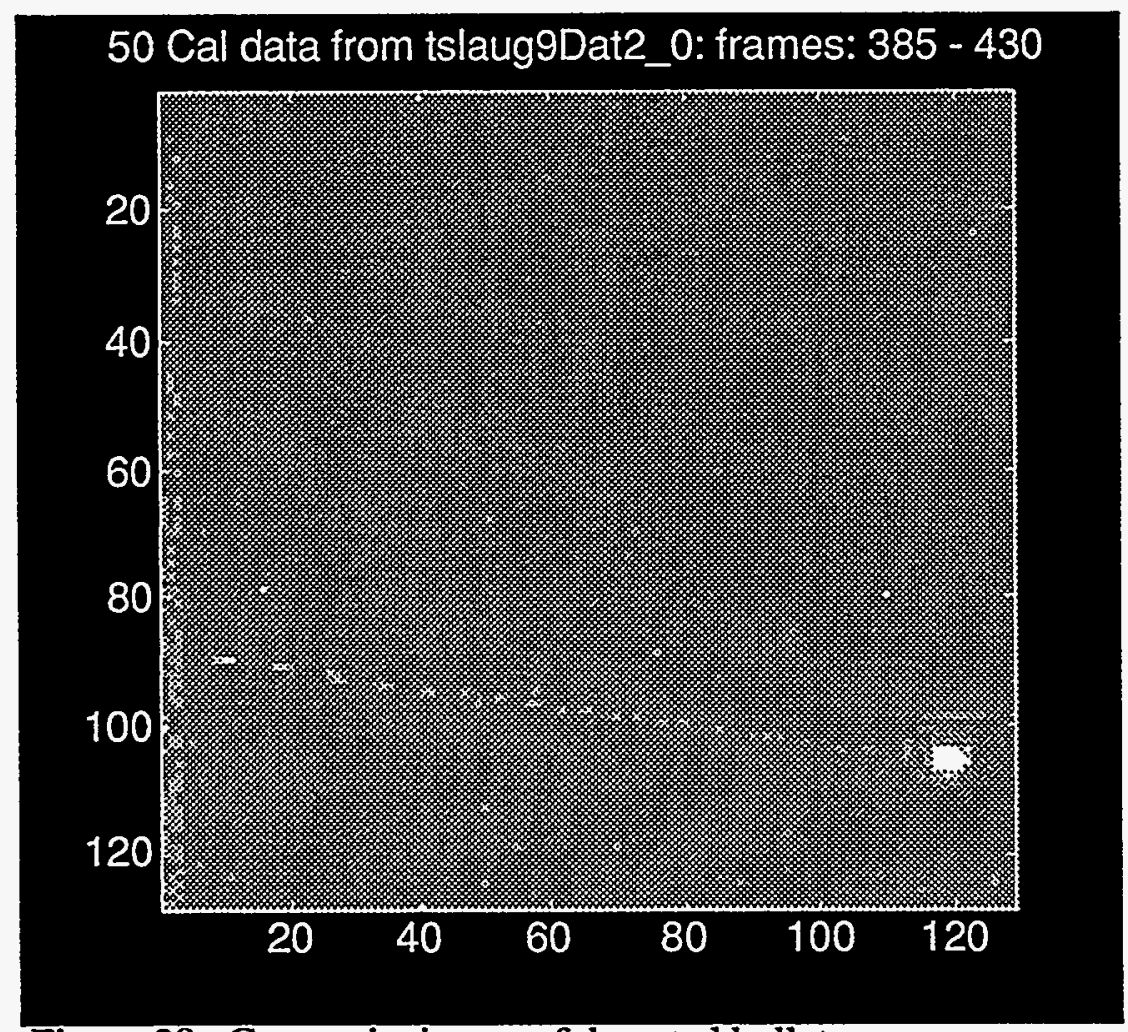

Figure 29 Composite image of detected bullets 


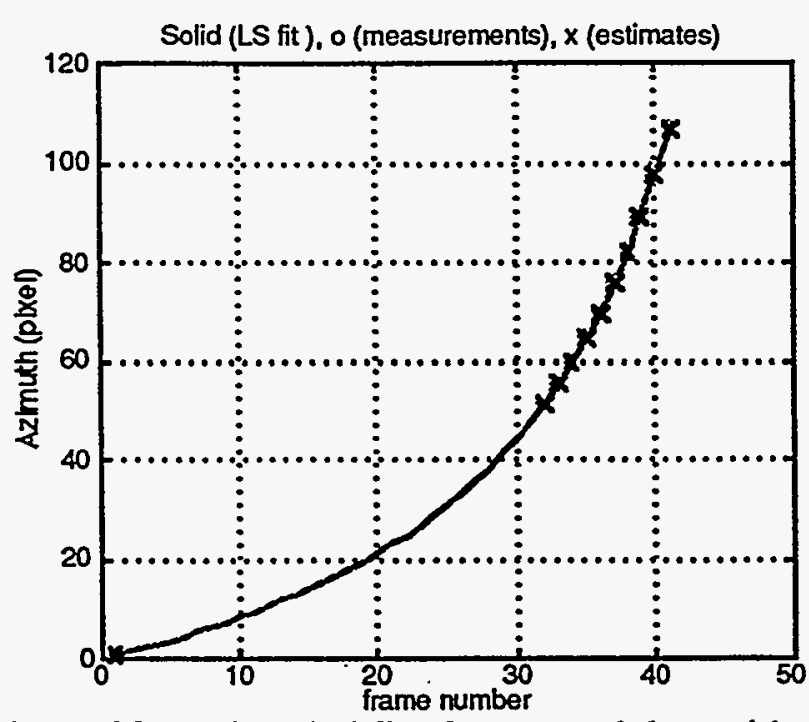

Figure 30 Azimuthal fit of measured data with time

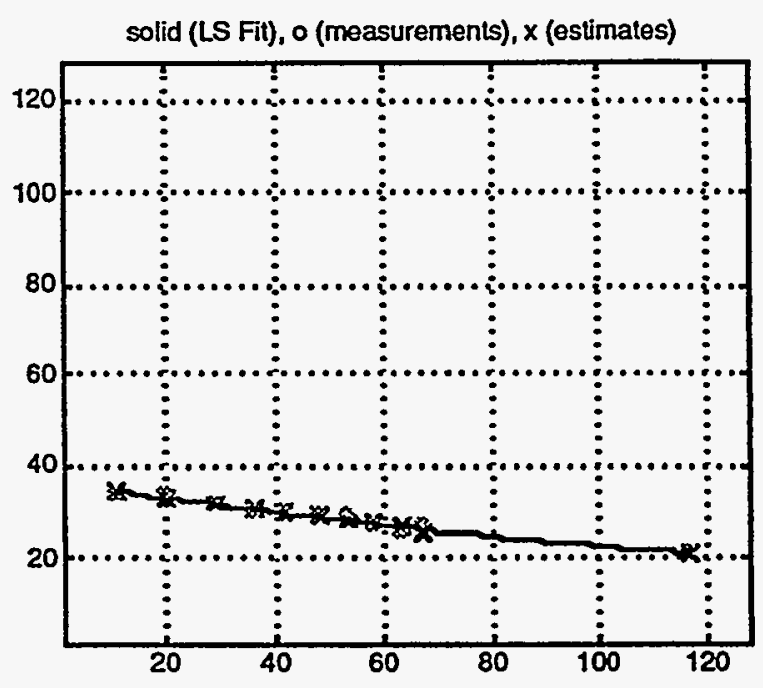

Figure 31 Resulting AZEL least squares fit on focal plane

Figure 30 shows the resulting least squares fit of actual azimuthal angles from the experiment. A very good fit is observed. The fit nicely connected the missing bullets. Experience shows that the ballistic model fit is superior than a polynomial fit, as the latter tends to curve around the missing bullet locations. Figure 31 compares the least squares fit trajectory from measured bullets to a reconstructed trajectory from the range geometry. Figure 32 provides an expanded plot of the reconstructed and actual measured trajectories on the FPA. Since we did not recorded the exact location of the bullet hole on the target, we could not precisely define the bullet track. However, we could construct two possible tracks, one for hitting the top of the target and the other for hitting the bottom of the target. Therefore if the bullet hit the target at all, it must be bounded between the two tracks. Using the least squares fit of the measured data, we

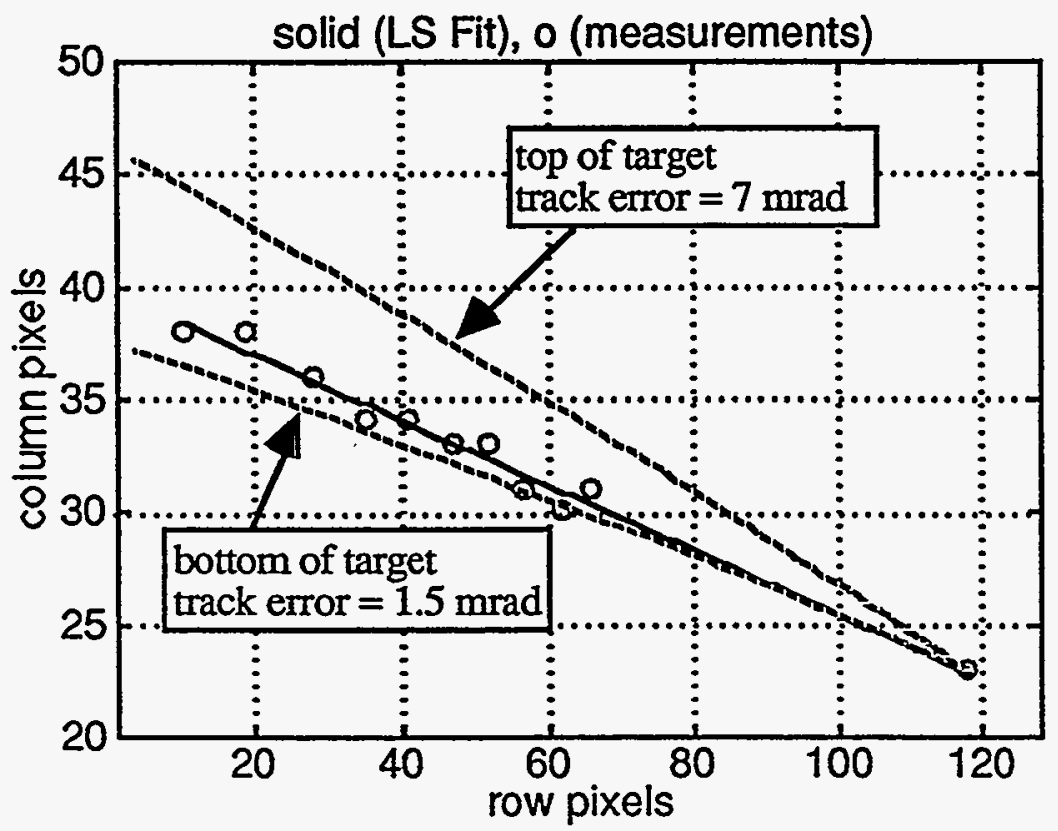

Figure 32 Least squares fit of measured data versus reconstructed path 


\section{Practical Considerations for Real-time Source Location}

We have demonstrated that our high-speed sensor and the real-time angle tracking software has the ability to accurately estimate a bullet's trajectory on a focal plane to $<100 \mu \mathrm{rad}$ for a 2 mrad IFOV sensor. While detecting as little as $25 \%$ of the theoretical total number of frames where the bullet is within the field-of-view of the sensor, we could reconstruct the track and also back-predict the locations of where the bullet should have been. For practical real-time applications in the field, one may want to know, for example: (1) How would one pick the initial state vector for precision angle tracking? (2) How to estimate the gun location if we don't see the muzzle flash? (3) What happens if there are multiple bullet in-flight simultaneously? and (4) What would be some of the design specifications for a practical sensor with high track accuracy?

First, picking the state vector for angular tracking is easy. Pick something reasonable and it will converge. The fact that we have an infinite number of solutions actually works to our advantage. It just needs to converge to any one solution and we will have established a robust angular track.

Second, in order to estimate the gun location without the muzzle flash, we can accomplish this in two ways. (1) Using no prior knowledge of the weapon, bound the gun position by extrapolating the angular track to where the angular rate goes to zero. The gun must be somewhere along the track between the zero-rate point and the first point where a bullet is detected. This is a small angle, usually just a few degrees. (2) Use assumed knowledge of a weapon type to bound its location. Since the estimate of the state vector is relatively insensitive to the ballistic coefficient error (a 6:1 reduction ) but almost one-to-one to the velocity error, we may simply assume a best guess for the ballistic parameter and bracket the muzzle velocity with probable minimum and maximum speeds based on experience with the shooter's probable weapon type. Then mapping the state vector back to the focal plane will bracket two possible locations on the angular track. The gun must be somewhere along the track between the locations for minimum and maximum muzzle velocity. This is a small angle, usually less than a degree. Our simulation results shown that with proper initial condition, the estimated time error of the muzzle flash could be within a few frames, and the angle could be less that 2 pixels or 4 mrad.

Third, multiple bullets would be handled by data association logic. Data association logic makes use of the fact that (1) each bullet track is a line on the focal plane, and (2) each bullet track has a distinct slope and intercept point. Thus for a design with $<200 \mu \mathrm{rad}$ track error, the system will be able to sort out tracks at $>200 \mu \mathrm{rad}$ apart.

Finally, based on our simulation study and augmented with experimental results, we believe a practical system that can meet, for example, a track error less than $200 \mu \mathrm{rad}$ will have specifications given by the following table.

Table 2. Preliminary Specifications for a Practical System

\begin{tabular}{|l|l|}
\hline Sensor Parameter & Specifications \\
\hline \hline Frame rate & $>200$ \\
\hline Field of view & $>60^{\circ}$ \\
\hline Pixel size & $2 \mathrm{mrad}$ \\
\hline \# of pixels & $512 \times 512$ \\
\hline pixel noise & $<1$ part in 4000 \\
\hline track error & $<200 \mu \mathrm{rad}$ \\
\hline
\end{tabular}




\section{Summary and Conclusions}

We have developed a methodology to estimate the bullet track as appearing on the focal plane and in actual geometric space. Our approach makes use of a simple ballistic model parameterized by a bullet's muzzle velocity and ballistic drag coefficient.

For AZEL tracking, we found that good angular track is obtained although the geometric parameters in range and shot-angle did not converge. There are multiple solutions to the angular least squares problem. However, anyone of those solutions will yield a good angular track. We also developed a metric called Effective Track Error to accurately characterize the track error between two bullet tracks on the FPA.

Using the effective track error as a metric, we investigated the AZEL track error sensitivity, via Monte-Carlo runs, to key design parameters such as range, shot-angle, angular noise, frame rates, and sensor field-of-view. Depending on the choice of design parameters and firing geometry, track errors in the range of $100 \mu \mathrm{rad}$ to $500 \mu \mathrm{rad}$ can realistically be obtained.

For ballistic tracking or source location estimation, we demonstrated that the system is indeed observable with knowledge of weapon parameters such as muzzle velocity and ballistic coefficient. We also conducted sensitivity analysis of the range and shot-angle variables to variation in muzzle velocity and ballistic coefficient. We found that the range parameter is almost linearly proportional to velocity error while the relation of shot-angle is not. Also, the percentage error of range and shot-angle to variation in the ballistic coefficient is significantly less sensitive - a factor of six in reduction is observed.

We have shown how the firing source location can be estimated under practical conditions, where little is known a priori about the weapon or shooter. The line-of-sight to the shooter can be bounded to a very small range along the apparent trajectory.

We have developed a detailed approach for bullet trajectory reconstruction from firing range data and knowledge of muzzle flash location on the camera FPA. The method developed a series of transformation matrices to relate any point in firing range coordinate to the camera coordinate.

We used the AZEL track estimator to analyze one particular shot conducted recently at LLNL's Site 300 facility. Our analysis of the measured data from the experiment shows that the observed track is within the upper and lower bounds of the bullet path. The deviation may be attributed to the movement of the shooter. A more accurate comparison with experiment can be obtained if the experiment also records the exact location of the bullet hole on the target.

Finally, we observed from experiments that accurate measurement of a muzzle flash location could be obtained during the contraction cycle of the flash. Thus a better understanding of the physics of the flash could lead to a consistent subpixel measurement of muzzle flash location. 


\section{Acknowledgments:}

The authors appreciate the assistance and contributions of the following individuals and organizations in making this work possible.

\section{U.S. Navy Special Boat Unit 11}

Jeff Frank

Monte Brandrup

Jeff Getz

Tom Tassinari

Wayne Luedtka

Bill Labiak

Robert Johnson

Jose E. Hernandez

Karl Krause

John Holzrichter

John Nuckolls

Bruce Tarter

Lowell Wood

Work performed under the auspices of the U.S. Department of Energy by Lawrence Livermore National Laboratory under contract W-7405-Eng-48. This study was supported by the Laboratory Directed Research and Development (LDRD) Director's Initiative Program on Weapon Neutralization. 


\section{References}

[1]. Joseph Hassab," Contact Localization and Motion Analysis in the Ocean Environment: A Perspective," IEEE Journal of Oceanic Engineering, Vol. OE-8. No.3, July 1983

[2]. V. J. Aidala and S.E. Hammel, "Utilization of Modified Polar Coordinates for Bearingsonly Tracking," IEEE Trans.on Automatic Control, Vol., AC-28, No.3, March 1983.

[3]. T. L. Song and J.L. Speyer,"A Stochastic Analysis of a Modified Gain Extended Kalman Filter with Applications to Estimation with Bearing Only Measurements," IEEE Trans. on Automatic Contyrol, Vol. AC-30, No. 10, October 1985.

[4]. Larry C. Ng and R.A. LaTourette," Equivalent bandwidth of a general class of polynomial smoothers," J. Acoustic. Soc. Am. 74(3), September 1983.

[5]. V.J. Aidala,"Kalman filter behavior in bearings-only tracking applications," IEEE Trans. Aerosp. Electron. System., Vol. AES-15, No. 1, pp 29-39, January 1979.

[6]. A. Gelb,"Applied Optimal Estimation," The M.I.T. Press, 1974

[7]. V.J. Aidala and S.C. Nardone,"Biased Estimation Properties of the Pseudolinear Tracking Filter," IEEE Trans. on Aerospace \& Elec. Systems, Vol. AES-18, No.4, July 1982.

[8]. F.J. Regan,"Re-entry Vehicle Dynamics," AIAA Education Series, 1983 


\section{Appendix A}

\section{Bullet Ballistic Model}

For our purposes of estimating the bullet track, a simple ballistic model will be utilized. Let the bullet at time $t$ has velocity $v(t)$ and at a distance $s(t)$ from the muzzle. We assume the principal force on the bullet is the aerodynamic drag on the order of hundreds of gs and the effect of gravity is negligible. If $\mathrm{m}$ is the mass of the bullet, then the equation of motion is given by:

$$
\begin{aligned}
\dot{\mathrm{V}} & =-\frac{1 / 2 \rho v^{2} C_{D} A}{m} \\
& =-\left(\frac{1 / 2 \rho}{m /\left(C_{D} A\right)}\right) \mathrm{v}^{2}=-\alpha \mathrm{v}^{2} ;
\end{aligned}
$$

where $\alpha$ is inversely proportional to the ballistic coefficient given by:

$$
\beta=\frac{m}{C_{D} A} .
$$

where $\mathrm{A}$ is the effective surface area and $C_{D}$ is the aerodynamic drag coefficient. Eq (A-1) can be solved to yield:

$$
\mathrm{v}(t)=\frac{\mathrm{v}_{o}}{1+\alpha \mathrm{v}_{0}\left(t-t_{0}\right)} ; \text { and } \mathrm{v}_{o}=\mathrm{v}\left(t_{o}\right)
$$

where $v_{0}$ is the muzzle velocity at $t=t_{0}$. Eq. $(A-3)$ can also be integrated to yield:

$$
s(t)=\ln \left(1+\alpha \mathrm{v}_{o}\left(t-t_{o}\right)\right) / \alpha .
$$

Eliminating the time variable in Eqs (A-3) and (A-4) yields:

$$
\mathrm{v}(t)=\mathrm{v}_{o} e^{-\alpha s(t)} .
$$

Thus the bullet velocity slows down exponentially with distance determined by the ballistic drag coefficient $\alpha$. We can also determine the ballistic drag coefficient from knowledge of bullet velocity and distance travel as a function of time from Eq. (A-5) as:

$$
\alpha=\frac{\ln \left(\frac{\mathrm{v}_{0}}{\mathrm{v}(t)}\right)}{s(t)} .
$$

For example, given a bullet with the following deceleration profile: $s(t)=[0,100,200]$ meters, and $v(t)=[975,850,735]$ meters $/ \mathrm{sec}$,

$$
\alpha=\ln (975 / 850) / 100=\ln (975 / 735) / 200=0.0014 .
$$




\section{Appendix B}

Computation of Partial Derivatives

Using the following notations:

$$
\begin{aligned}
\mathrm{s}_{\mathrm{k}} & =\text { distance bullet at time } t_{\mathrm{k}}, \\
\mathrm{x}_{\mathrm{k}} & =\mathrm{x} \text { coordinate of bullet at time } \mathrm{t}_{\mathrm{k}}, \\
\mathrm{y}_{\mathrm{k}} & =\mathrm{y} \text { coordinate of bullet at time } t_{\mathrm{k}} \text {, and } \\
r_{\mathrm{k}} & =\sqrt{x_{k}^{2}+y_{k}^{2}},
\end{aligned}
$$

and the relation

$$
\begin{aligned}
h & =\tan ^{-1}\left(\frac{x_{k}}{y_{k}}\right) \\
& =\tan ^{-1}\left(\frac{s_{k} \sin (\varphi)-x_{0}}{y_{0}-s_{k} \cos (\varphi)}\right),
\end{aligned}
$$

then the following derivatives can be written as:

$$
\begin{aligned}
& \frac{\partial h}{\partial y_{0}}=-\frac{s_{k} \sin \varphi}{r_{k}^{2}} \\
& \frac{\partial h}{\partial v_{0}}=\left[\frac{y_{k} \sin \varphi+x_{k} \cos \varphi}{r_{k}^{2}}\right]\left[\frac{\left(t_{k}-t_{o}\right)}{1+\alpha v_{o}\left(t_{k}-t_{o}\right)}\right] \\
& \frac{\partial h}{\partial \alpha}=\left[\frac{x_{k} \cos \varphi+y_{k} \sin \varphi}{r_{k}^{2}}\right]\left[\frac{\partial s_{k}}{\partial \alpha}\right],
\end{aligned}
$$

where

$$
\frac{\partial s_{k}}{\partial \alpha}=\frac{1}{\alpha^{2}}\left[\frac{\alpha \nu_{o} t_{k}}{1+\alpha \nu_{o} t_{k}}-\ln \left(1+\alpha v_{o}\left(t_{k}-t_{o}\right)\right)\right]
$$

and finally

$$
\frac{\partial h}{\partial \varphi}=\frac{s_{k}}{r_{k}^{2}}\left[y_{k} \cos \varphi-x_{k} \sin \varphi\right]
$$




\section{Appendix C}

Survey Map at LLNL Site 300 Firing Range

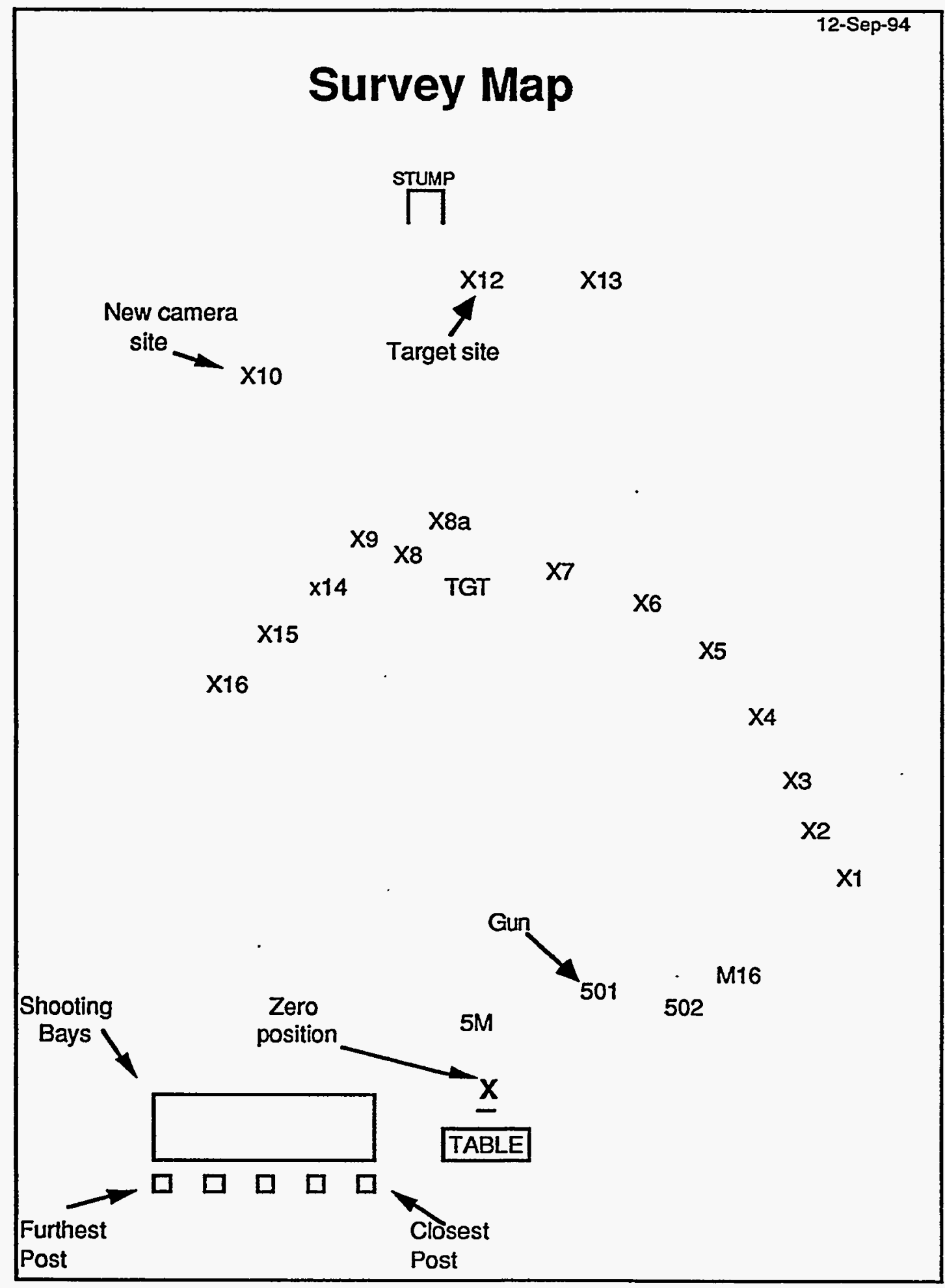




\section{Surveyed Points at Site $\mathbf{3 0 0}$ Shooting Range}

\begin{tabular}{|c|c|c|c|c|c|c|}
\hline position & distance & gle & height & $\mathbf{x}$ & $\mathbf{Y}$ & $\mathbf{z}$ \\
\hline & 80.21 & 20.40 & 5.46 & 75.18 & -27.96 & 5.46 \\
\hline $\mathrm{X} 2$ & 82.54 & 17.78 & & 78.60 & -25.20 & 5.46 \\
\hline $\mathrm{x} 3$ & 86.26 & 14.36 & & 83.56 & -21.39 & 5.46 \\
\hline $\mathbf{X} 4$ & 89.56 & 11.31 & & 87.82 & -17.56 & 5.46 \\
\hline X5 & 94.89 & 7.94 & 5.49 & 93.98 & -13.11 & 5.49 \\
\hline $\mathrm{X} 6$ & 98.33 & 5.49 & 5.39 & 97.88 & -9.41 & 5.39 \\
\hline $\mathrm{X7}$ & 96.33 & 2.88 & 5.06 & 96.21 & -4.84 & 5.06 \\
\hline X8 & 99.56 & 357.55 & 4.17 & 99.47 & 4.26 & 4.17 \\
\hline X8a & 105.9 & 357.08 & 4.09 & 105.76 & 5.39 & 4.09 \\
\hline X9 & 100.04 & 353.43 & 3.41 & 99.38 & 11.45 & 3.41 \\
\hline$\times 10$ & 216.34 & 356.89 & 10.74 & 216.02 & 11.74 & 10.74 \\
\hline X11 & 229.48 & 358.67 & 12.91 & 229.42 & 5.33 & 12.91 \\
\hline $\mathbf{X 1 2}$ & 225.38 & 0.28 & 15.73 & 225.38 & -1.10 & 15.73 \\
\hline X13 & 222.50 & 5.15 & 15.27 & 221.60 & -19.97 & 15.27 \\
\hline X14 & 98.36 & 350.18 & 3.00 & 96.92 & 16.78 & 3.00 \\
\hline X15 & 95.33 & 346.61 & 2.59 & 92.74 & 22.08 & 2.59 \\
\hline X16 & 92.37 & 343.59 & 2.31 & 88.61 & 26.10 & 2.31 \\
\hline $100 \mathrm{~m}$ tgt & 91.20 & 0.00 & 4.92 & 91.20 & 0.00 & 4.92 \\
\hline stump & 245.53 & 358.34 & 16.52 & 245.43 & 7.11 & 16.52 \\
\hline 1st light & 5.11 & 0.00 & N/A & 5.11 & 0.00 & \\
\hline Table & 3.30 & 180.00 & & -3.30 & 0.00 & \\
\hline rifle barrel & 2.90 & 180.00 & & -2.90 & 0.00 & \\
\hline nearest pole & $1.90 \mathrm{~N} / \mathrm{A}$ & & N/A & -3.30 & 1.90 & \\
\hline 2nd pole & $4.90 \mathrm{~N} / \mathrm{A}$ & & N/A & -3.30 & 4.90 & \\
\hline 3rd pole & $7.95 \mathrm{~N} / \mathrm{A}$ & & N/A & -3.30 & 7.95 & \\
\hline 4th pole & $11.04 \mathrm{~N} / \mathrm{A}$ & & N/A & -3.30 & 11.04 & \\
\hline furthest pole & $13.95 \mathrm{~N} / \mathrm{A}$ & & N/A & -3.30 & 13.95 & \\
\hline 50 cal1 & 7.96 & 10.90 & & 7.82 & -1.51 & 0 \\
\hline $50 \mathrm{cal2}$ & 8.77 & 26.30 & & 7.86 & -3.89 & \\
\hline M60 & 10.20 & 39.70 & & 7.85 & -6.52 & \\
\hline
\end{tabular}




\section{Appendix D}

Bullet Trajectory Reconstruction from Firing Range Data

Let $\mathbf{G}\left(\mathrm{xg}, \mathrm{yg}_{\mathrm{g}} \mathrm{zg}\right)$ and $\mathbf{T}\left(\mathrm{x}_{\mathrm{t}}, \mathrm{yt}_{\mathrm{t}}, \mathrm{z}_{\mathrm{t}}\right)$ be the vectors from the origin of $\mathrm{XYZ}$ system to the gun and the target respectively (see Figures $24 \& 25$ of the report), then a unit vector along the bullet track is given by:

$$
\mathbf{u}_{t_{8}}\left(u_{x}, u_{y}, u_{z}\right)=\frac{\mathbf{T}-\mathbf{G}}{|\mathbf{T}-\mathbf{G}|}
$$

Let $P(x, y, z)$ be a vector to any point on the bullet track in the $X Y Z$ system. Then we must have the following relation:

$$
P(x, y, z)-G\left(x_{g}, y_{g}, z_{g}\right)=s u_{t g},
$$

where $s$ is a scalar ranges from $s=0$ to $s=D$, the distance between the gun and the target. Solving Eq.(D-2) results in a method to generate the bullet trajectory as follows:

$$
\left[\begin{array}{l}
x \\
y \\
z
\end{array}\right]=\left[\begin{array}{l}
x_{g} \\
y_{g} \\
z_{g}
\end{array}\right]+s\left[\begin{array}{l}
u_{x} \\
u_{y} \\
u_{z}
\end{array}\right]-\left[\begin{array}{c}
0 \\
0 \\
0.5 * g * t^{2}
\end{array}\right],
$$

where we have included the effect of gravity on the $\mathrm{z}$ component. For a $200 \mathrm{~ms}$ flight time, the contribution from gravity is less than $20 \mathrm{~cm}$. Note that assuming a knowledge of the ballistic coefficient and muzzle velocity, the distance the bullet travels, $\mathrm{s}$, at time $t$ can be related ( See Appendix A). We next address the coordinate transformation problem.

As shown in Figure 24, we fix a rectangular coordinate system $\left(X_{1}, Y_{1}, Z_{1}\right)$ to the left bottom corner of the FPA looking from the back of the camera such that the initial camera orientation is given by $\mathrm{X}_{1}=\mathrm{Y}, \mathrm{Y}_{1}=-\mathrm{X}$, and $\mathrm{Z}_{1}=\mathbf{Z}$, or in matrix form:

$$
\left[\begin{array}{l}
x_{1} \\
y_{1} \\
z_{1}
\end{array}\right]=\left[\begin{array}{ccc}
0 & 1 & 0 \\
-1 & 0 & 0 \\
0 & 0 & 1
\end{array}\right]\left[\begin{array}{l}
x \\
y \\
z
\end{array}\right]=A\left[\begin{array}{l}
x \\
y \\
z
\end{array}\right]
$$

where A represents the transformation matrix. Now let $C\left(x_{S}, y_{S}, z_{S}\right)$ be the vector to the measured sensor (or camera) location (i.e., origin of the $\mathbf{X}_{1}, \mathbf{Y}_{1}, \mathbf{Z}_{1}$ system), we can express all vectors w.r.t. this initial camera system as: 


$$
\left[\begin{array}{l}
x_{1} \\
y_{1} \\
z_{1}
\end{array}\right]=A\left[\begin{array}{l}
x-x_{s} \\
y-y_{s} \\
z-z_{s}
\end{array}\right]
$$

Figure 25 shows the a reference vector from sensor to muzzle flash expressed in initial camera coordinate system. Let this vector be denoted as $R\left(R_{x 1}, R_{y 1}, R_{z 1}\right)$, then w.r.t the $Y_{1}$ axis, the reference vector is pointed at a direction with azimuth, $\theta_{R}$, and elevation, $\phi_{R}$. Both angles can be computed from:

$$
\left[\begin{array}{l}
\theta_{R} \\
\phi_{R}
\end{array}\right]=\left[\begin{array}{c}
\tan ^{-1}\left(x_{1} / y_{1}\right) \\
\tan ^{-1}\left(z_{1} / \sqrt{x_{1}^{2}+y_{1}^{2}}\right)
\end{array}\right] .
$$

We can align the $Y_{1}$ axis along the reference vector by performing a negative rotation about $Z_{1}$ through an angle, $\theta_{R}$, and a positive rotation about $X_{1}$ through an angle, $\phi_{R}$. The resulting transformation to an intermediate system $\left(\mathbf{X}_{2}, \mathbf{Y}_{2}, \mathbf{Z}_{2}\right)$ is given by:

$$
\left[\begin{array}{l}
x_{2} \\
y_{2} \\
z_{2}
\end{array}\right]=\operatorname{Rot}_{x}\left(\phi_{R}\right) \operatorname{Rot}_{z}\left(-\theta_{R}\right)\left[\begin{array}{l}
x_{1} \\
y_{1} \\
z_{1}
\end{array}\right] \text {, }
$$

where Rot( )s are Euler rotation angles given by [8]:

$$
\operatorname{Rot}_{z}(\theta)=\left[\begin{array}{ccc}
\cos (\theta) & \sin (\theta) & 0 \\
-\sin (\theta) & \cos (\theta) & 0 \\
0 & 0 & 1
\end{array}\right]
$$

and

$$
\operatorname{Rot}_{x}(\phi)=\left[\begin{array}{ccc}
1 & 0 & 0 \\
0 & \cos (\phi) & \sin (\phi) \\
0 & -\sin (\phi) & \cos (\phi)
\end{array}\right]
$$

Finally if the measured muzzle flash appearing on the FPA is at azimuth $\theta_{\mathrm{m}}$, and elevation $\phi_{\mathrm{m}}$, we can accomplish that by a positive rotation about $Z_{2}$ through an angle $\theta_{\mathrm{m}}$ and a negative rotation about $\mathbf{X}_{2}$ through an angle $\phi_{\mathrm{m}}$. The final system $\left(\mathbf{X}_{\mathrm{c}}, \mathbf{Y}_{\mathrm{c}}, \mathbf{Z}_{\mathrm{c}}\right)$ is the reconstructed 
camera orientation. Therefore, collecting all the necessary Euler angle rotations performed, we can transform any vectors in the $(\mathbf{X}, \mathbf{Y}, \mathbf{Z})$ system to the camera system from the relation:

$$
\begin{aligned}
{\left[\begin{array}{l}
x_{c} \\
y_{c} \\
z_{c}
\end{array}\right] } & =\operatorname{Rot}_{x}\left(-\phi_{m}\right) \operatorname{Rot}_{z}\left(\theta_{m}\right)\left[\begin{array}{l}
x_{2} \\
y_{2} \\
z_{2}
\end{array}\right] \\
& =\operatorname{Rot}_{x}\left(-\phi_{m}\right) \operatorname{Rot}_{z}\left(\theta_{m}\right) \operatorname{Rot}_{x}\left(\phi_{R}\right) \operatorname{Rot}_{z}\left(\theta_{R}\right) A\left[\begin{array}{l}
x \\
y \\
z
\end{array}\right] .
\end{aligned}
$$

